This article may not exactly replicate the final version published in the APA journal. It is not the copy of record.

\title{
Are goal states represented during kinematic imitation?
}

\author{
Geoff G. Cole ${ }^{1}$, Mark A. Atkinson ${ }^{2}$, Antonia D. C. D’Souza ${ }^{1}$, Timothy Welsh ${ }^{3}$, \& Paul A. Skarratt ${ }^{4}$ \\ 1. Centre for Brain Science, University of Essex. \\ 2. Institute of Education, University of London. \\ 3. Faculty of Kinesiology \& Physical Education, University of Toronto. \\ 4. Department of Psychology, University of Hull.
}

Running head: Kinematics and goals.

Address for correspondence

Geoff G. Cole

Centre for Brain Science

University of Essex

Wivenhoe Park

Colchester

CO4 3 SQ

E-mail: ggcole@essex.ac.uk

Tel: 01206872331

Fax: 01206873801 


\begin{abstract}
A number of studies have shown that observation of another person's actions can modulate one's own actions such as when two individuals cooperate in order to complete a joint task. However, little is known about whether or not direct matching of specific movements is modulated by the goals of the actions observed. In a series of seven experiments we employed an action observation paradigm in which two coactors sat opposite each other and took turns to reach out to targets presented on a shared workspace. Importantly, co-actors performed either the same goal at the reached-to location or a different goal. Although results consistently showed that the reaching action of one individual slows the observer's reaching action to the same spatial location, the effect was not modulated according to the adopted goals of coactors. These findings challenge the notion that the processes involved in the imitation of specific movements code for the action goals of those movements.
\end{abstract}

\title{
Public significance of the work
}

It is well known that we, as humans, often imitate the actions of other people. Interestingly, this can include the imitation of specific motor patterns. For instance, if we see another person reach out with their right arm this will speed up the time it takes us to reach out with our own right arm. However, it is not yet known whether this imitation effect is dependent upon the observer and the person being observed having the same reason (or 'goal') for performing the action. For example, if the person being observed reached out to pick up a coffee cup will they still speed up the observer's action if they reach out to pick up a pen to write with? The present research assessed this question and found that imitation effects are not influenced by goals.

Key words: Attention; movement compatibility; IOR; Social; Joint action; imitation 


\section{General introduction}

The past decade or so has seen a growth in the number of studies examining the cognitive processes that occur when an individual observes another person's action. Such action observation studies are often placed within the context of perceptuo-motor models (e.g., Hommel, 2009; Jeannerod \& Frak, 1999; Knoblich \& Sebanz, 2006; Prinz, 1997) in which, rather than being separate, perception and action are said to share cognitive representations. One assumption that follows from these models is that an action by one individual activates an equivalent internal motor representation in an observer. Consequently, observing a specific manual movement can influence an observer's own preparation for that same movement; what Iacoboni et al. (1999) refer to as the direct matching hypothesis.

A typical effect was reported by Liepelt, Von Cramon, and Brass, (2008; see also, Brass, Bekkering, Wohlschläger, \& Prinz, 2000; Kilner, Paulignan, \& Blakemore, 2003). In their experiment, participants viewed images of a hand topographically oriented in the same position as their own response hand. As one of the fingers was lifted on the stimulus hand, a target also appeared requiring the participants to raise either their middle or index finger. The important manipulation was the compatibility between the finger raised on the stimulus hand and that lifted by the participants. A compatible trial was one in which, for instance, the index finger on the photograph was raised and the response also required the index finger to be raised. An incompatible trial by contrast was one in which the index finger on the photograph was raised but the response required the middle finger to be raised. Results showed that response time (RT) was shorter for compatible trials compared with incompatible trials. As described above, this finding suggests that the 
mechanisms responsible for planning specific movements are sensitive to features of the observed movement.

A further example that has generated considerable interest, and that is central to the present work, illustrates how one person's reaching action can modulate the reaching action of an observer (Atkinson, Skarratt, Simpson, \& Cole, 2014; Cole, Skarratt, \& Billing, 2012; Cole, Wright, Doneva, \& Skarratt., 2015; Doneva \& Cole, 2014; Hayes, Hansen, \& Elliott, 2010; Lyons, Weeks, \& Elliott, 2013; Ondobaka, de Lange, Newman-Norlund, Wiemers, \& Bekkering, 2012; Skarratt, Cole, \& Kingstone., 2010; Welsh et al., 2007; Welsh et al., 2007; Welsh, Manzone, \& McDougall, 2014; Welsh, McDougall, \& Weeks, 2009; Welsh, Ray, Weeks, Dewey, \& Elliott, 2009). In the basic paradigm, first described by Welsh et al. (2005), two people are seated opposite across a table and take turns to reach out to targets appearing to the left or right on a workspace between them (see Figure 1). Welsh et al. reported that RT increased for targets appearing at the same location as a coactor's previous response. The authors suggested that this same-location slowing effect was due to a combination of action-perception representations and mechanisms giving rise to inhibition of return (IOR; Posner \& Cohen, 1984). Specifically, Welsh et al. argued that observing a co-actor's response activates the corresponding response codes in the observer, with slowed reaching to the same location reflecting the inhibition accrued from returning attention and repeating responses ${ }^{1}$. This interpretation is supported by the finding that when a single individual makes two consecutive responses to the same location, RT in the second response increases relative to a response made to a different location (Maylor \& Hockey, 1985; Tremblay, Welsh, \& Elliott, 2005; Welsh et al. 2005). 
One of the central theoretical questions associated with action observation studies is whether the mechanisms that represent action and motor responses also represent the goals of those actions. In a broad sense this is clearly the case in highly coordinated actions such as when two individuals move a piece of furniture. As well as representing the overall goal, each co-actor needs to represent the other's actions with respect to immediate sub-goals (e.g., avoiding a door frame). This kind of goal hierarchy is central to the goal directed theory of imitation (Bekkering, Wohlschläger, \& Gattis, 2000), which makes the distinction between imitation processes that represent end-point actions (i.e., goals) and those that represent motor commands or 'kinematics primitives' (i.e., specific movements). The theory suggests that mechanisms associated with the former have primacy over mechanisms associated with the latter, an idea for which there is growing support. For example, when observers are asked to imitate an action, they tend to copy the goal of the action rather than the kinematics of the movements that brought about the goal (Bekkering, et al., 2000). Furthermore, Longo, Kosobud, and Bertenthal (2008) suggest that representation of actions at the level of goals is an automatic 'default response'. Moreover, the mirror neuron system, hypothesised as one of the mechanisms subserving action understanding (di Pellegrino, Fadiga, Gallese, \& Rizzolatti, 1992), is thought to primarily represent goals rather than body movement (e.g., Rizzolatti, Fabbri-Destro \& Cattaneo, 2008).

Since goals and intentions are more important in everyday joint actions compared with specific movements, the dominance of goals over kinematics has clear functional utility. Indeed, during goal imitation the mechanisms associated with kinematic imitation may be somewhat redundant or even inhibited. This is because a single goal often can be achieved via several different kinematic routes, while a single 
kinematic route can eventuate into several different outcomes. The focus of the present study is to examine whether the underlying goals of an actor influence the initiation of specific movements of an observer. To be clear, our experiments are not concerned with the question of whether goals are important for modulating an observer's action, or even modulating the perception of those actions, they evidently are (i.e., Bekkering, et al., 2000; and see Hudson, Nicholson, Ellis, \& Bach, 2016, for perception). Rather, our experiments examined whether goals modulate imitative movements, i.e., those that occur as a result of direct matching. A number of experiments do suggest that goals influence kinematics. For example, Wohlschläger and Bekkering, (2002) reported that finger movement responses were sensitive to whether the observed fingers of a model were directed towards a goal or not (see also Bouquet, Shipley, Capa, \& Marshall, 2010). Using a similar finger movement paradigm, Liepelt et al., (2008) showed that the degree of imitative matching was modulated according to whether the model intentionally moved their fingers (i.e., adopted a goal), as opposed to having them moved by a mechanical device. However, these goals tended to be relatively simple in nature (e.g., the volitional pressing of a button), whereas goals requiring greater dexterity to accomplish them have shown no influence on kinematics. In one such example, Cole et al. (2012) used a variant of the Welsh et al. (2005) arm reaching paradigm described above. In each of three experiments, participants reached to either the same or opposite location to their partner, but then performed one of two end-point actions. For instance, in one experiment participants were required to either touch a corner of a diamond or pretend to grip the diamond as if they were going to pick it up. In another experiment, participants reached to a pencil and either wrote a digit on a pad or used its opposite end to erase a digit. Importantly, on half of the trials both actors performed the same 
task and therefore adopted the same goal as each other (e.g., both wrote a digit), whilst on the other half they performed a different task (e.g., one wrote, one erased). Results from all three experiments showed that the presence and magnitude of the basic same-location slowing effect was independent of the co-actors' goal states. That is, the magnitude of the differences between RTs to targets presented at the same and different locations was not affected by the (in)compatibility of the goals of the task. These findings suggest that the mechanisms that represent observed kinematics do not code for goals (or that subsequent action planning and initiation was insensitive to goals and the coding of movement kinematics was the primary influence). Furthermore, one other direct matching study has shown an effect that is opposite to what might be expected if goals are represented (i.e., a significantly smaller effect for actions that are compatible with those observed (Chiavarino, Bugiani, Grandi, \& Colle, 2013).

In contrast to the findings of Cole et al. (2012), Ondobaka et al. (2012), also employing the Welsh et al. paradigm, reported that observed goal states do modulate specific movements during action observation. In their experiment, the participant and a confederate co-actor were each presented with two playing cards, one located on the left and one on the right of the workspace. The participant was required to reach to the playing card with the higher (or lower) value based on the value selected by the co-actor on the previous trial. On one block of trials, if the co-actor reached to the higher card, the participant was asked to do the same on their turn (i.e., reach to their higher card). In contrast, on another block, the participant was asked to do the opposite with respect to the co-actor. For instance, if the co-actor reached for the higher card the participant was asked to reach to the lower card. Thus, as with Cole et al., the two co-actors either had a congruent or incongruent action goal and reached to 
either the same or a different spatial location. Unlike Cole et al. however, Ondobaka et al. found that RTs were modulated by the congruency of goals adopted by the coactors, i.e., the same-location slowing effect occurred only when both actors performed the same task. In a follow-up study using the same arm reaching paradigm, Ondobaka, Newman-Norlund, de Lange, and Bekkering (2013) reported that observers spontaneously represent the goals of their co-actor. Given the benefits associated with an ability to predict others' actions, such a finding is consistent with Sebanz, Knoblich, and Prinz’s (2005) argument that “one may find oneself unable not to represent others' actions and intentions” (p1245).

Given the contrasting findings, the aim of the present study was to examine whether the mechanisms associated with the generation of imitative movements represent the goals of the action observed. Interestingly, there are several methodological differences between the studies of Cole et al. (2012) and Ondobaka et al. (2012; 2013) may point to the conditions under which goal representation influences kinematics. For example, in the experiments of Cole et al., the goal adopted by an observer's co-actor was independent of the observer's task. By contrast, in the experiment of Ondobaka et al. (2012), participants were required to monitor their co-actor's action on a trial-by-trial basis. Despite Ondobaka et al.'s (2013) later claim concerning spontaneous goal recognition, this difference suggests that actions may code for goals only when the movements of a co-actor are relevant to one's own. Furthermore, the Cole et al. experiments manipulated goals in terms of the action to be performed at the end-point of the reaching response (e.g., whether to draw or erase a digit). By contrast, Ondobaka et al. manipulated goals at the start point (to which stimulus participants should reach). Hence, it may be that observers' 
kinematic mechanisms represent only the selection goals of a co-actor rather than their end-point goal.

In the present experiments, participants alternated reaching responses to the same or opposite location to their partner. This served to generate the basic arm reaching phenomenon reported by Welsh et al. (2005) and Skarratt et al. (2010). We then assessed the effect of goals on movements by manipulating the goal congruity of co-actors, such that they performed either the same or a different goal. The different experiments examined a number of ways in which goals might influence kinematic imitation, including task relevancy (Experiment 1), start- versus end-point action (Experiment 2), location of response (Experiment 3), top-down versus bottom-up processes (Experiment 4), and cooperation on a shared task (Experiment 5). Although these experiments consistently yielded an action observation effect, the effect was largely independent of the goal congruency of participants. Experiments 6 and 7 were direct replications of the designs employed by Ondobaka et al. (2012; 2013). Results again showed no goal-influenced effect. Taken together, these findings suggest that when individuals perform a similar kinematic action to one just observed, the processes mediating action observation and action planning are insensitive to the underlying goals.

\section{Experiment 1}

The work of Folk, Remington, and Johnston (1992) revealed the importance of task relevance on the propensity with which a stimulus can modulate attention. For instance, a nominally 'task irrelevant' colour cue is more likely to capture attention when the target being searched for is defined by colour (Folk et al., 1992). With respect to joint action, studies demonstrate that knowledge of a co-actor's intention can influence an observer's action planning. For example, Sun and Thomas (2013) 
showed that target detection was improved for targets that appeared adjacent to a coactor's hands, but only when they were relevant to accomplishing a shared goal. In the action observation experiments of Cole et al. (2012), participants undertook a number of different blocks in which they either performed the same task as their coactor or a different task. Although participants were aware of the task being performed by their partner, and indeed could see this task throughout the trials, the task was not relevant to their own - that is, participants did not need to monitor their partner's actions. By contrast, in the Ondobaka et al. (2012) experiment (although not Ondobaka et al. 2013), the required goal for participants on each trial was determined by the actions of their co-actor. In other words, the participant knew what to do on any particular trial only by observing what their co-actor had just done on the previous trial (e.g., reach to a high card). This methodological difference, as with the Sun and Thomas study, suggests that the action observation system may only represent or prioritize a co-actor's goals when their actions are directly relevant to the observer's goal. We therefore closely replicated Experiment 1 of Cole et al. (2012) with the exception that we manipulated whether a co-actor's task was relevant or irrelevant to the participant's task. On all trials, participants were presented with a single diamond figure target on one side of the display and required to perform one of two possible end-point responses on the object. On task irrelevant blocks they were told which of the two tasks their co-actor would be performing but that it was incidental to their own task, whilst on task relevant blocks they were told that they should copy the action that their co-actor had just performed on each trial.

\section{Method}

Participants. Cole et al. (2012) and Ondobaka et al. (2013) both employed 16 participants in their experiments. We therefore decided, a priori, to employ 16 
participants in all the seven experiments we report here. The exception to this was Experiment 2 in which 18 participants were included. This experiment was undertaken some time before the others when we did not fully weight the consideration of participant number in our recruitment. Each experiment, including the one referred to in the discussion to Experiment 7, was composed of a different set of participants who were naive to its purpose. In all experiments, with the exception of Experiment 4, the participant's co-actor was a confederate whose responses were not analysed.

Stimuli and apparatus. In the action observation paradigm of Welsh et al. (2005), coactors inevitably sit in slightly different positions with respect to the workspace and stimuli. We therefore provide all measurements in mm instead of visual angle. A black fixation cross $\left(0.4 \mathrm{~cd} / \mathrm{m}^{2}\right.$ measured on-screen $)$ was presented in the centre against a uniform white background $\left(66.4 \mathrm{~cd} / \mathrm{m}^{2}\right)$. The participants were instructed to reach to a black diamond $\left(0.4 \mathrm{~cd} / \mathrm{m}^{2}\right)$ that measured $27 \mathrm{~mm}$ along each side. This diamond would appear $175 \mathrm{~mm}$ from the fixation cross, as measured from its middle. Stimuli were presented on a 22" LCD monitor built into a table positioned between each co-actor and a Keytec touchscreen was located over the monitor. Participants sat with their chests approximately $240 \mathrm{~mm}$ from a 'home' button and the monitor was $740 \mathrm{~mm}$ from the floor. Participants made a response by moving their right hand from the home button to the target object. The interval between target presentation and the release of the home button was recorded and this was used as RT. Many previous papers that have used the present paradigm have also recorded and reported arm/hand movement times (e.g., Skarratt et al. 2010; Welsh et al. 2005). None, however, has found any effects, demonstrating that the basic phenomenon reflects processes occurring prior to an action being initiated. We therefore do not report movement 
times in any of the present experiments. The exception to this is Experiment 7 in which we performed a replication of a previous study that did include movement time in the analysis. An RM Pentium PC running custom software controlled stimulus generation and the recording of responses.

Design and procedure. We employed a within-participants $2 \times 2 \times 2$ design. The first factor manipulated target location relative to the previous trial (repeated or opposite). A repeated trial was one in which the target occurred at the same location whilst an opposite trial was one in which the target occurred on the opposite side. Recall that differences in these two levels generate the basic action observation effect in which RTs are usually longer on repeated (same) location trials than on different (opposite) location trials. The second factor manipulated the goal each co-actor performed with respect to each other (same or different). The third factor manipulated task relevancy. Either the confederate co-actor's task was relevant to the participant or irrelevant. Participants took part in four blocks of trials crossing task relevancy (relevant, irrelevant) with goals (same, different) which were counterbalanced across participants in a Latin square. Only the target location factor was mixed within blocks. Participants (and the co-actor) performed one of two goals; they were asked to either "touch the point of the diamond nearest to you" or "pretend to grip the diamond with your thumb and index finger as if you are going to pick it up”. In the two task irrelevant blocks, the co-actor's goal was not relevant to the goal of the participant and they were informed of this. At the beginning of the two task irrelevant blocks, participants were told that they should perform the touch task on every trial. On one of these blocks the co-actor would perform the same goal on every trial (i.e., touch) whilst on the other block he would perform a different goal (i.e., grip). Participants were told which of the two goals their co-actor would be performing at the beginning 
of each block. On the two task relevant blocks, participants were told to attend to what the co-actor did on each trial and either repeat that action (i.e., goal) or perform the other action. Therefore, unlike the two task irrelevant blocks, on these two blocks the co-actor performed a different goal trial-by-trial; a goal that was directly relevant to the participant's task. On the (task relevant) trials in which the confederate co-actor responded, he was informed which response to make by the word 'grip' or 'touch' that appeared on screen in a position visible only to him. Participants were asked to alternate responses with the co-actor, respond as quickly and as accurately as possible, and to return their hand to the home button after a response. The program generated random target locations using the same constraints as in Welsh et al. (2005). Thus, the frequency with which repeated and opposite trials occurred was the same as was the number of left and right targets. Furthermore, no target appeared on the same side more than four times in a row. Five-hundred ms elapsed between response completion and the appearance of the next target. Four blocks of trials were presented, each being composed of 209 trials; 105 for the confederate co-actor and 104 trials for the participant. Participants were also given 16 practice trials. All experiments reported here were approved by the psychology department's ethics committee at the University of Essex. Participant consent was obtained through a system in which individuals chose one of a number of experiments to perform as part of a psychology undergraduate 'methods' module. The exception to this was the experiment reported in the discussion section of Experiment 7 where participants chose to undertake the experiment in return for £8. These participants were both undergraduates and postgraduates at the University of Essex who replied to an advertisement. 


\section{Results and discussion}

In addition to frequentialist null hypothesis significance testing, we conducted a series of Bayesian analyses for each experiment using the free software JASP version 0.7.5.6. (Love et al., 2015; Morey \& Rouder, 2011). Using effect size distributions, the software computes Bayes factors that compare against two competing hypotheses by assessing the relative probability of the observed data according to each. A model was calculated for each main effect and interaction across all experiments using the default prior estimate, which treats each model as equally probable. This Bayesian approach allowed us to compare the models for goal congruency and action observation against the null hypothesis, as well as the interaction model against the null hypothesis. In addition, following Wagenmakers (2007), we compared models of single or additive main effects against interaction models, which is not possible under traditional frequentialist methods. Thus we could evaluate the relative evidence for an influence of goals on action observation, against the evidence for the alternative, where these factors were independent and/or additive. In addition we report analyses comparing Bayes factors for interaction models, with main effects models as alternative hypotheses. In interpreting the strength of evidence in favour of a particular alternative hypothesis, we adopt the approach of Rafferty (1995).

Outliers (RTs beyond \pm 2 SDs for each participant’s condition mean) accounted for $4.2 \%$ of responses in Experiment 1 and were omitted from further analysis. Figure 2 shows mean RTs. An ANOVA with target location (repeated or opposite), goal (same or different), and task relevancy (relevant or irrelevant) as within-participants factors revealed a significant main effect of target location, $F(1,15)=32.6$, $p<.001$, $\eta_{p}^{2}=.685, B F_{10}=3.25 \mathrm{e}+6$, and task relevancy, $\mathrm{F}(1,15)=8.1, \mathrm{p}<.01, \eta_{p}^{2}=.35$ 
$B F_{10}=187172.12$, but no main effect of goal, $\mathrm{F}(1,15)=.05, \mathrm{p}>.82, \eta_{p}^{2} .003, B F_{10}$ $=0.20$. The interaction between goal and task relevancy was not significant, $F(1,15)$ $=.01, \mathrm{p}>.9, \eta_{p}^{2} .001, B F_{10}=8531.88$, neither was the interaction between task relevancy and target location, $\mathrm{F}(1,15)=.18, \mathrm{p}=.62, \eta_{p}^{2}=.01, B F_{10}=1.23 \mathrm{e}+6$. There was, however, a significant goal by target location interaction, $F(1,15)=4.8$, $\mathrm{p}$ $=.04, \eta_{p}^{2}=.24, B F_{10}=163138.05$, and a significant three-way interaction, $F(1,15)=$ 6.3, $\mathrm{p}=.02, \eta_{p}^{2}=.30, B F_{10}=5158.88$. The Bayesian analysis compared the additive main effects model containing target location and task relevancy, with a model specifying an interaction between goal and target location. This analysis revealed preference for the additive main effects model over the interaction model between these factors, $B F_{10}=3.99$, corresponding to weak evidence in favour of the alternative hypothesis that there was a main effect of task relevancy and target location only over an interaction. However the three-way interaction model was preferred over this additive model, $B F_{10}=4195.03$, which corresponds to very strong evidence in favour of a three-way interaction between target location, goal and task relevancy. The Bayesian analysis thus supports the results of the ANOVA, namely that although there is no evidence in favour of an interaction between goal and target location, a three-way interaction between these factors and task relevancy was supported.

The first important aspect of these results is the presence of the action observation effect reported by Welsh et al. (2005). Participants had longer RTs when responding to the target at the same position as the co-actor's previous response than to the opposite location. This finding replicates the many earlier reports of the phenomenon (e.g., Ondobaka et al. 2012; Skarratt et al. 2010; Welsh et al. 2005; 
2007). Most importantly, however, was the finding that task relevancy influenced the degree to which the effect was modulated by the goals of the co-actor: when the coactor's goal was relevant to the participant's task, the action observation effect was larger when actors had a different goal compared to when they had the same goal. By contrast, when the co-actor's goal was not relevant to the participant's task, the action observation effect did not differ according to whether the same goal was performed or not. This was confirmed by two separate $2 \times 2$ follow-up ANOVAs (on the relevant and irrelevant condition means) which revealed a significant interaction, $F(1,15)=$ 12.0, $\mathrm{p}=.003, \eta_{p}^{2}=.44, B F_{10}=5.67$ when the co-actors' task was relevant but no such interaction when the co-actors task was irrelevant, , $\mathrm{F}(1,15)=.5, \mathrm{p}=.493, \eta_{p}^{2}=$ $.03, B F_{10}=1.30$. Importantly, this additional analysis and results are opposite to what the goals-based theory of direct matching predicts, as well as the results obtained by Ondobaka et al; one should expect a larger, not smaller, action observation effect when co-actors have the same goal. The fact that the present results do show a goalbased effect demonstrates that the present experimental design is sensitive enough to reveal the influence of goals. This finding is therefore consistent with the results of Chavarino et al. (2013) who found that an action observation effect was smaller when a participant's action was compatible with that of an observed action. It is not, however, consistent with the notion that movements are facilitated when the observed and performed action match with respect to goal.

\section{Experiment 2}

An intention or goal can be defined in many different ways. Indeed, it is not clear how one can most accurately define a goal. In the Cole et al. (2012) experiments the goals were defined, and thus differed, in terms of end-point action; that is, the action participants performed once they had reached the target location (e.g., write or 
erase a digit). However, the basis on which they selected a response location was always the same (localize a target stimulus). By contrast, in Ondobaka et al. (2012; 2013), the end-point action was always the same (touch the screen) but participants selected their response location according to the selection rule of their co-actor (choose a high/low value card). Selection and action are semi-independent processes subserved by different brain mechanisms. For instance, neurological patients can often select an item from distractors but are unable to appropriately act upon them (e.g., Sirigu, Cohen, Duhamel, Pillon, Dubois, \& Agid, 1995). Similarly, some patients are able to effectively act upon a stimulus but are not able to make an appropriate discrimination of the stimulus responded to (e.g., Milner \& Goodale, 1995). This difference in the results of Cole et al. and Ondobaka et al. could be due to their operational definitions of goals as respectively end-point and start point. The principal aim of the current experiment was to assess this distinction.

Following Ondobaka et al.'s operational definition of a goal, participants undertook the basic arm reaching task in which goals were defined by selection. Coactors were required to reach out and touch one of two letters forming a pair on the left or right side of the display. The participants' goal was to either reach to the same letter as their co-actor or the different letter. As the goal was defined at the point of selection, the location of the response - and the response itself - remained the same.

\section{Method}

Stimuli and apparatus. All aspects of the experimental set-up were as described in Experiment 1 with the following exceptions. Targets were either the letter $\mathrm{S}$ or $\mathrm{H}$ (measuring $20 \mathrm{~mm}$ in height and $14 \mathrm{~mm}$ in width) and were presented immediately adjacent to each other (see Figure 3). 
Design and procedure. We employed a within-participants $2 \times 2$ design. As with Experiment 1, the first factor manipulated target location (repeated or opposite), whilst the second factor manipulated the goal congruency of the two participants (same or different). Participants undertook two blocks of trials in which they were instructed to reach out and touch the letter ' $S$ ' on every trial (thus, their co-actor's response was always task irrelevant). In one of these blocks, the confederate co-actor would also reach to the letter S (i.e., same goal) whilst in another block he reached to the letter H (i.e., different goal).

\section{Results and discussion}

Outliers were defined as in Experiment 1 and accounted for 3.9\% of responses which were omitted from further analysis. Figure 4 shows mean RTs. An ANOVA with target location (repeated or opposite) and goal (same or different) as withinparticipants factors revealed a significant main effect of target location, $F(1,15)=$ 36.6, $\mathrm{p}<.001, \eta_{p}^{2}=.71, B F_{10}=43.59$, with RTs on repeated target trials being longer than those on opposite trials. Importantly, there was no main effect of goal, $\mathrm{F}(1,15)=.49, \mathrm{p}=.61, \eta_{p}^{2}=.02, B F_{10}=0.32$, and the interaction between goal and location was not significant, $\mathrm{F}(1,15)=3.0, \mathrm{p}=.11, \eta_{p}^{2}=.17, B F_{10}=5.86$. Bayesian analysis compared the interaction model between target location and goal with a model specifying a main effect of goal only. This revealed a preference for the main effect model, $B F_{10}=2.47$, representing weak evidence in favour of the alternative hypothesis of a main effect of target location only over an interaction with goal. Overall, these data once again reveal the basic same-location slowing effect. However, even though goals were defined on the basis of a selection rule rather than as end-point actions, RTs were independent of the goal states of others. 


\section{Experiment 3}

One of the most fundamental properties of attentional selection is the distinction between selection based on spatial location and selection based on object representations. Duncan (1984) originally showed that when participants are asked to make simple perceptual judgments, performance is better if the decisions concern a single object compared to different objects, even when the latter all occur within the perimeter of the single object and thus occupy the same amount of space. Similarly, attention is known to move more rapidly between two locations if they are grouped to form a single object (Egly, Driver, \& Rafal, 1994) and the inhibition accrued during IOR can travel with an object as it moves from one location to another (Jordan \& Tipper, 1998; Tipper, Jordan \& Weaver, 1999). Furthermore, the present action observation effect is also known to be sensitive to locations and objects. For instance, Atkinson et al. (2014) demonstrated that perceptually grouping a non-responded-to location with a location that a co-actor just reached to induces the same-location slowing effect.

In the experiments of Cole et al. (2012), objects (and locations) were shared between the co-actors: when a participant reached to the same side of the display as a co-actor's previous response, it was always to the same object (e.g., Figure 1). By contrast, in Ondobaka et al.'s (2012; 2013) studies, the stimuli to which participants reached were located in the four corner segments of the array; thus, different objects and different locations. This methodological difference raises the possibility that actions may represent goals only when co-actors act on different but adjacent objects and not when actors reach to the same object/location. In the present experiment therefore, we closely replicated the procedure of Cole et al. (2012; Experiment 1) with the exception that co-actors responded to a different object when reaching to the 
same side as the previous response (see Figure 5). This change alone, however, would not enable us to differentiate between any goal-based action effect based on an object representation and one based on a spatial representation; responding to two separated objects invariably means that co-actors respond to two different locations. We therefore designed the experiment such that on half the trials co-actors reached to different locations contained within the same object. This was achieved through grouping the two locations in a manner commonly employed in many object-based attention experiments (e.g., Crundall, Cole, \& Galpin, 2007; Egly et al. 1994; see the present Figure 5).

\section{Method}

Stimuli and apparatus. All aspects of the experimental set-up were as described previously with the following exceptions. Participants were presented with two black diamonds $\left(0.4 \mathrm{~cd} / \mathrm{m}^{2}, 27 \mathrm{~mm}\right.$ along each side), both located on one side of the display. These locations would occur $92 \mathrm{~mm}$ from each other and $182 \mathrm{~mm}$ from the fixation cross, as measured from their middle. In the grouped conditions, they were placed within a grey rectangle measuring $155 \mathrm{~mm}$ by $47 \mathrm{~mm}$.

Design and procedure. We employed a within-participants $2 \times 2 \times 2$ design. The first factor manipulated target location (repeated or opposite), the second goal (same or different), and the third grouping (grouped or separate). Participants undertook four blocks of trials: 1) objects grouped, same goal, 2) objects grouped, different goal, 3) objects separate, same goal, 4) objects separate, different goal. Whilst participants always performed the same goal in all four blocks, being instructed to "touch the point of the diamond nearest to you”, their confederate co-actor performed either this touching task or pantomimed the gripping task of Experiment 1. The co-actor's 
response was always incidental to the participant's task. All other aspects of the design and procedure were as described previously.

\section{Results and discussion}

Outliers, defined as above, accounted for 3.8\% of responses and were omitted from further analysis. Figure 6 shows mean RTs. A within-participants factorial ANOVA revealed a significant main effect of target location, $F(1,15)=28.8, p<$ $.001, \eta_{p}^{2}=.66, B F_{10}=439.85$, but no significant effect of grouping, $\mathrm{F}(1,15)=.1, \mathrm{p}$ $>.74, \eta_{p}^{2} .001, B F_{10}=0.21$, or goal, $\mathrm{F}(1,15)=.66, \mathrm{p}>.42, \eta_{p}^{2}=.043, B F_{10}=.29$ The interactions between goal and grouping, $\mathrm{F}(1,15)=1.20, \mathrm{p}=.18 \eta_{p}^{2}=.12, B F_{10}$ $=0.04$, location and grouping, $\mathrm{F}(1,15)=.17, \mathrm{p}=.21 \eta_{p}^{2}=.10, B F_{10}=19.49$ and location and goal $\mathrm{F}(1,15)=.44, \mathrm{p}=.52 \eta_{p}^{2}=1.03, B F_{10}=37.61$ were not significant. There was also no significant location $\mathrm{x}$ goal $\mathrm{x}$ grouping interaction $\mathrm{F}(1$, 15) $=1.49, \mathrm{p}=.24 \eta_{p}^{2}=0.09, B F_{10}=0.66$. Bayesian analysis compared the interaction model between goal and target location, with the model for a main effect of target location only. This analysis revealed a preference for the main effect model, $B F_{10}=663.42$, corresponding to decisive evidence in favour of the alternative hypothesis of a main effect of target location against an interaction between target location and goal. Overall, these results show that, although an action observation effect was present, it was not influenced by the goals of the co-actors. This absence of a goal-based effect occurred irrespective of whether co-actors reached to the same location or different location, or if the targets were part of the same object or not. This finding therefore supports the emerging conclusion that the kinematics of the action have the primary influence, whereas the coding of the goals of the action have a minimal, if any, influence. 


\section{Experiment 4}

In the experiments of Cole et al. (2012), and the present Experiments 1-3, participants directed their actions to a single left or right location by a peripheral target. The response location was therefore determined in a 'bottom-up' manner by an attention-capturing target event. By contrast, in the experiments of Ondobaka et al. (2012; 2013), the response location was determined by a 'top-down' process in which participants selected the location based on some feature of two stimuli (e.g., which card has the higher value?). The discrepant results by Cole et al. and Ondobaka et al. may therefore reflect the fundamental difference between exogenous and endogenous processes (see for instance, Itti \& Koch, 2000). The implication is that actions may be sensitive to goals when a movement is made towards a location based on endogenous attention but not when the location is determined by an exogenous cue. Indeed, one might argue that goals are primarily a top-down entity as, by their very definition, they represent prospective intentionality - that is, an individual's immediate or future plans. A further and related issue concerning the top-down/bottom-up distinction made between the experiments of Cole et al. and Ondobaka et al. was the requirement for participants to attend respectively to one or both sides of the display. The imperative in Cole et al. was for participants to react to the onset of a single target on any given trial, while those in Ondobaka et al. were asked to make a judgement between two stimuli. Indeed, previous evidence has shown that the arm movement phenomenon is reduced in tasks where participants must discriminate between two objects on different sides of the display (Atkinson et al., 2014). In the present experiment therefore, participants were required to monitor the stimuli presented on both sides and respond to one, as in Ondobaka et al. (2012; 2013). Specifically, two objects were presented, one on either side, and participants reached to one of them. 


\section{Method}

Stimuli and apparatus. All aspects of these were as described previously with the following exceptions. Participants were presented with two objects, one located on either side of the display. These differed either in colour or in form. In the colour discrimination blocks, the two objects were two squares (14 mm along each side) each positioned $175 \mathrm{~mm}$ from the fixation cross, as measured from their middle (see Figure 1). One square was red $\left(10.6 \mathrm{~cd} / \mathrm{m}^{2}\right)$, the other was green $\left(50.6 \mathrm{~cd} / \mathrm{m}^{2}\right)$. In the form discrimination blocks, one object was a solid black square whilst the other was a solid black circle (both $0 \mathrm{~cd} / \mathrm{m}^{2}$ ). The size of the squares was as reported above. The circle was $8 \mathrm{~mm}$ in diameter. Unlike all other experiments reported here, the two co-actors were experimental participants, rather than one participant and one confederate coactor as in Experiments 1-3.

Design and procedure. We employed a within-participants $2 \times 2 \times 2$ design with target location (repeated or opposite), goal (same or different), and task (colour or form) as factors. With respect to the last factor, participants were required to make discrimination responses, either responding to one of the two coloured squares, or to one of the two shapes. This instruction necessitated participants to attend to both sides of the array before selecting the correct one. All participants took part in eight blocks of trials with each block requiring a different or same task with respect to their partner. Specifically, participant pairs undertook eight variations of the task conditions. For the colour discrimination blocks: 1) both reached to the red target, 2) both reached to the green target, 3) Participant A reached to the red target, Participant $\mathrm{B}$ to the green target, 4) Participant A reached to the green target, Participant B to the red target. For the form discrimination blocks: 5) both reached to the circle, 6) both reached to the square, 7) Participant A reached to the circle, Participant B to the 
square, 8) Participant A reached to the square, Participant B to the circle. Each coactors' task was always irrelevant to that of their partners', although each person was always made aware of their partner's response goal. The presentation order of the colour and form discrimination blocks was counterbalanced and the goal blocks were presented in a random order.

\section{Results and discussion}

There were $3.2 \%$ of responses identified as outliers and omitted from further analysis. Figure 7 shows mean RTs for each of the eight conditions. An ANOVA with target location, task, and goal as within-participants factors revealed a significant main effect of target location, $\mathrm{F}(1,17)=8.20, \mathrm{p}<0.02, \eta_{p}^{2}=.33, B F_{10}=0.39$, but neither the main effects of task, $\mathrm{F}(1,17)=.63, \mathrm{p}=.44, \eta_{p}^{2}=.04, B F_{10}=.58$, nor goal, $\mathrm{F}(1,17)=1.67, \mathrm{p}=.21, \eta_{p}^{2}=.036, B F_{10}=0.70$, were significant. There was no interaction between task and goal, $\mathrm{F}(1,17)=1.62, \mathrm{p}=.44, \eta_{p}^{2}=.04, B F_{10}=.11$, task and location, $\mathrm{F}(1,17)=.01, \mathrm{p}=.93, \eta_{p}^{2}<.001, B F_{10}=0.05$, nor goal and location, $\mathrm{F}(1,17)=.26, \mathrm{p}=.619, \eta_{p}^{2}=.02, B F_{10}=0.07$. Finally there was no significant threeway interaction, $\mathrm{F}(1,17)=.034, \mathrm{p}=.86, \eta_{p}^{2}=.002, B F_{10}=9.04 \mathrm{e}-4$. Bayesian analysis compared a model for the interaction between goal and target location, with the model for a main effect of target location only. This revealed a preference for the main effect model, $B F_{10}=8.84$, corresponding to moderate evidence in favour of the alternative hypothesis of an effect of target location only over an interaction. Comparison of the three-way interaction model with the main effect of target location only, demonstrated preference for the main effect model, $B F_{10}=491.78$, corresponding to very strong evidence in favour of the alternative hypothesis that 
there was a main effect of target location over a three-way interaction. Overall, these data reveal that the action observation effect occurs irrespective of whether co-actors respond to a peripheral exogenous stimulus or discriminate between two stimuli. This finding in turn does not support the notion that the goal of the observed action influences the movement planning of the observer.

\section{Experiment 5}

As already suggested, one of the challenges in examining (and therefore manipulating) goal states in the laboratory is that goals can be represented hierarchically across any number of levels, ranging from specific to the general, from proximal to distal. For instance, whereas experimenters might define a goal as reaching to a red or a green square, the participants' goal might simply be to participate in a laboratory experiment in exchange for payment or course credit. Furthermore, as Paternotte (2014) has pointed out, "There exist many competing philosophical definitions of joint action and no clear criteria to decide between them; so far the search for definitions has by and large been a semantical enterprise rather than an empirical one” (p. 103). Paternotte goes on to suggest that most definitions of joint action imply the collective adoption of common goals. In its broadest sense, all participants in joint action experiments adopt the common goal of participating in a joint action task. In Experiment 5, then, we sought to define and manipulate a goal in the most consensual and unequivocal way achievable in a laboratory setting. To that end, co-actors again undertook the reaching paradigm but were required to actively collaborate (or not) on a shared task across an entire block of trials, with successful completion dependent on cooperation. Specifically, participants were required to reach out, pick up a pen, and either jointly complete a dot-to-dot drawing with their co-actor or perform this task without the assistance of their partner. Thus, in the 
former condition cooperation was required in order to complete the shared goal whilst in the latter there was no cooperation and the goal could not be completed. The design was therefore based on a common purpose or intention (i.e., goal) that could be accomplished only through explicit cooperation.

\section{Method}

All aspects of these methods were as described previously with the following exceptions. As with our other experiments, participants were asked to reach to a left or right location depending on the position of a target. Once they had done so, they were then required to pick up a pen resting on a pad of paper, and draw one small section of a house as one does in a child's dot-to-dot drawing book (see Figure 8). They were informed that they could choose any section to draw on each trial. This differed from the common dot-to-dot drawings in which numbers are placed adjacent to the dots suggesting a particular drawing sequence. There were 103 dots on each drawing. This meant that both houses (i.e., left and right) would be completed when both co-actors (i.e., participant and confederate) performed the drawing task but not when only the participant did this task. The special cues were solid black circles (0 $\mathrm{cd} / \mathrm{m}^{2}$, diameter $8 \mathrm{~mm}$ ) appearing for $100 \mathrm{~ms}$ and positioned $175 \mathrm{~mm}$ to the left or right of a centrally located fixation point. The pens were located on the two pads measuring $24 \mathrm{~cm}$ long, $15 \mathrm{~cm}$ wide and $4 \mathrm{~cm}$ high. Their height meant that both were level with the touchscreen.

Design and procedure. We again employed a $2 \times 2$ design with the first factor manipulating target location and the second factor manipulating goal. All aspects of the procedure were as described previously with the exception that participants were asked to reach out to the target location and attempt to complete the dot-to-dot drawing. On one block of trials they were told that they would need to cooperate with 
the co-actor to achieve this goal whilst on another block they were told that the coactor would not be cooperating; instead they would be drawing a number 7 on the pad on each trial. The presentation order of the two blocks was counterbalanced.

\section{Results and discussion}

Outliers accounted for $3.6 \%$ of all responses and were omitted from further analysis. Figure 9 shows mean RTs. An ANOVA revealed a significant main effect of

target location, $\mathrm{F}(1,15)=8.3, \mathrm{p}<.05, \eta_{p}^{2}=.36, B F_{10}=0.42$, and a significant main effect of goal, $\mathrm{F}(1,15)=19.0, \mathrm{p}<.001, \eta_{p}^{2}=.56, B F_{10}=2238066.36$. The interaction, however, was not significant, $\mathrm{F}(1,15)=2.4, \mathrm{p}=.14, \eta_{p}^{2}=.14, B F_{10}=$ 111926.80. The Bayesian analysis compared an interaction model between target location and goal, against the alternative hypothesis of an additive main effects model containing these factors. This revealed preference for the additive main effects model, $B F_{10}=1.46$, corresponding to weak evidence in favour the alternative hypothesis of an additive effect of goal and target location over an interaction. Thus, despite the fact that co-actors were required to explicitly cooperate, these data therefore once again demonstrate that movement planning in a joint action context is not influenced by goals.

\section{Experiment 6}

Experiments 1-5 teased apart the differences in the methods of previous experiments in order to examine when goals influence kinematics and when they do not. We have found however that imitative movements are not influenced by goal states. Experiments 6 and 7 therefore represented a slight shift in focus by performing 
direct replications of Ondobaka et al. (2012; 2013). Experiment 7 will also examine an alternative account to the imitation explanation of the basic same-location slowing effect.

In Experiment 6, we replicated ${ }^{2}$ the procedure of Ondobaka et al. (2013) in which the authors argued for 'spontaneous goal recognition' (cf. Ondobaka et al., 2012, in which goal recognition was an explicit component of the task). In their 2013 experiment, four blocks of trials were presented in which 'movement-control' and 'action-control' was manipulated. In the former, participants were instructed to reach out to the same location on every trial. Thus, on one block they were required to always reach to the left side whilst on another block they always reached to the right. In the latter (i.e., action-control), participants were instructed to reach to a particular relative value card on every trial. Thus, on one block they were required to always reach to the higher value card whilst on another block they reached to the lower value card. Their confederate co-actor, by contrast, reached to either high or low on every trial of each block (indicated by a cue). This meant that on half the trials within each block, participants happened to reach to the same relative value card as their co-actor (e.g., both reached 'high'), whilst on the other half they happened to reach to a different relative value card (e.g., co-actor reached 'low', participant reached 'high’). Ondobaka et al. found that the same-location slowing effect occurred only when the participant and co-actor happened to respond to the same relative value card, i.e., had the same goal, an effect which itself occurred only in the movement-control condition. That is, when participants guided their behaviour based on a physical movement. Ondobaka et al. argued that these findings demonstrate that participants spontaneously recognise a co-actor's goal.

\section{Method}


All aspects of the Method were as described previously and closely followed Ondobaka et al. (2013). Thus, on each trial, four 'spade’ cards $(4.5 \mathrm{~cm}$ x $6.5 \mathrm{~cm})$ were presented faced down. The confederate co-actor was presented with a visual cue (either the letter ' $\mathrm{H}$ ' for 'High' or 'L' for 'Low') informing him of which card should be selected on that trial. The cue was presented in the middle of the display and was visible only to the co-actor. After a variable delay of between 0.5 and $2.5 \mathrm{~s}$, the cards on the confederate's side of the display were revealed and he reached to the higher or the lower value card as per his instruction. The execution of his response caused the cards on the participant's side of the display to be revealed and the participant then reached out and selected the appropriate card/location. Both actors returned their hand to the home button immediately after touching the screen and the next trial was initiated when the participant had completed this move. Four blocks of trials were presented in which the participant performed one of four goals: 1) reach to the left hand card, 2) reach to the right hand card, 3) reach to the high value card, and 4) reach to the low value card. The first two blocks were therefore the 'movement-control' conditions of Ondobaka et al. whilst the last two were the 'action-control' conditions. The participants were not informed as to whether their co-actor was instructed to select the high or low card. The additional aspects of the cards outlined by Ondobaka et al. were also employed here. Thus, the four cards were always different; the higher/lower card appeared on the left and right with equal probability; the numerical value of the two cards always differed by one; the card selected by the participant was independent of the one selected by the co-actor; and 'catch' trials were included in which participants were instructed not to respond, but which required them to scan all the cards. Catch trials occurred on $23 \%$ of trials and were denoted by the presence of a card with a value of ' 2 ' appearing on the participant's or the co-actor's side with 
equal probability. Each block consisted of 104 trials and their presentation order counterbalanced. A $2 \times 2 \times 2$ design was employed in which target location, goal, and task were manipulated. Note that in order to maintain consistency with all other experiments reported here, we refer to target location as being repeated or opposite. This contrasts with Ondobaka et al. who, in suggesting that same-location RT slowing is due to movement compatibility, refer to 'movement-congruent' and 'movementincongruent' as opposed to opposite and repeated, respectively.

\section{Results and discussion}

A total of $3.1 \%$ of responses were identified as outliers and were omitted from further analysis. Figure 10 shows mean RTs. An ANOVA with target location, goal, and task as within-participants factors revealed a significant main effect of task, $F(1$, 15) $=60.9, \mathrm{p}<.001, \eta_{p}^{2}=.8, B F_{10}=4.32+\mathrm{e} 31$, though neither the main effects of location, $\mathrm{F}(1,15)=.98, \mathrm{p}=.34, \eta_{p}^{2}=.06, B F_{10}=.19$, nor goal, $\mathrm{F}(1,15)=.03, \mathrm{p}$ $=.86, \eta_{p}^{2}=.002, B F_{10}=.16$, were significant. The effect of task revealed that RTs were shorter in the movement-control than in the action-control conditions. Across goal $\mathrm{x}$ task, $\mathrm{F}(1,15)=1.59, \mathrm{p}=.23, \eta_{p}^{2}=.10, B F_{10}=2.33 \mathrm{e}+30$, goal $\mathrm{x}$ location, $\mathrm{F}(1$, $15)=.08, \mathrm{p}=.79, \eta_{p}^{2},<.001, B F_{10}=.01$ and location $\mathrm{x}$ task, $\mathrm{F}(1,15)=.70, \mathrm{p}=.42$, $\eta_{p}^{2}<.05, B F_{10}=2.45 \mathrm{e}+30$, none of the interaction effects were significant. There was additionally no three-way interaction, $\mathrm{F}(1,15)=2.26, \mathrm{p}=.02, \eta_{p}^{2}=.13, B F_{10}=$ 1.42e+28. To further maintain consistency with Ondobaka et al. (2013), we also examined the two levels of task (movement control and action control) separately in two 2 x 2 ANOVAs. With respect to movement control, there was no effect of goal, $\mathrm{F}(1,15)=.5, \mathrm{p}>.4, \eta_{p}^{2}=.03, B F_{10}=.29$, nor target location, $\mathrm{F}(1,15)=1.0, \mathrm{p}>.32$, 
$\eta_{p}^{2}<.06, B F_{10}=0.52$. There was, however, a marginally significant goal $\mathrm{x}$ target location interaction, $\mathrm{F}(1,15)=4.5, \mathrm{p}=.051, \eta_{p}^{2}=.23, B F_{10}=.13$, suggesting that the reaching effect is larger in the different goal conditions. With respect to action control, again neither the effect of goal, $\mathrm{F}(1,15)=.55, \mathrm{p}=.47, \eta_{p}^{2}=.035, B F_{10}=$ 0.33, nor target location, $\mathrm{F}(1,15)=.04, \mathrm{p}=.85, \eta_{p}^{2}=.003, B F_{10}=.31$ were significant and there was no interaction between these factors, $\mathrm{F}(1,15)=0.56, \mathrm{p}=$ .47, $\eta_{p}^{2}=.04, B F_{10}=0.39$. As none of the Bayes factors favoured an individual effect, additive effects or an interaction model over the null hypothesis model, no further model comparison was undertaken for either the movement control or action control levels of task. Overall, the present results do not replicate those previously reported by Ondobaka et al. (2013). Recall that they observed the present action observation effect only when the confederate co-actor's goal coincided with that of the participants. The authors suggested that these findings revealed spontaneous goal recognition. We, however, found no evidence for spontaneous recognition of goal congruency. Indeed, the reaching phenomenon was larger in the movement-control condition when the co-actor and participants performed different goals. Although an abundance of evidence does show that goal inference can occur spontaneously (Csibra, et al., 1999; Hassin et al., 2005; Van der Cruyssen, et al., 2009), our results suggest that such automatic inference does not occur at the level at which specific movements are represented. In sum, these results concur with Cole et al. (2012), and our current experiments: action observation does not appear to represent the goals of co-actors or at minimum, the coding of the kinematics of the action has the primary influence relative to goal coding. 


\section{Experiment 7}

The aims of Experiment 7 were twofold. First, we sought to examine why the basic same-location slowing effect occurs, and second, to replicate the procedure of Ondobaka et al. (2012) in which the recognition of the partner's goal was an explicit part of the task (cf. Ondobaka et al., 2013). This protocol can be viewed as the more liberal of the two experiments reported by Ondobaka and colleagues (i.e., Ondobaka et al., 2012; 2013) because the goals effect occurred when participants were required to perform the goal just seen rather than the goal being computed spontaneously. Recall from the Introduction that the confederate co-actor in Ondobaka et al. (2012) reached to the higher or lower of two cards and the participant was required to perform the same goal (in one block) and the different goal (in another block).

With respect to the primary aim of examining why the basic phenomenon occurs, the majority of authors have placed the effect within the context of inhibitory processes (e.g., Cole et al, 2012; Skarratt et al, 2010; Welsh et al, 2005). For instance, Welsh et al. (2005) showed that in the same way that participants are slower to aim two consecutive responses to the same spatial location, they are also slower when repeating the response of their co-actor. Welsh et al. argued that the same response codes are activated when performing or observing an action, thus giving rise to the same inhibitory aftereffect. Whilst Welsh et al. argue that the effect is due to action co-representation mechanisms they do not place it within the context of direct matching. They do however additionally suggest that the effect is mediated by the mirror neuron system (di Pellegrino, et al. 1992). This network is thought to become active both during action execution and when the same action is observed. As Welsh et al. (2007) stated, "We hypothesize that the activation of the mirror neuron system during the observation of the response mimicked the activity associated with the 
actual response” (p. 955). It is this mimicking that was said to induce IOR in the observer. Ondobaka, et al. (2012) however, placed the phenomenon within the context of movement compatibility. Thus, the authors suggested that a reach to the right hand target by co-actor A will facilitate a reach by co-actor B to their own right hand side. This facilitation results in reduced RTs to targets appearing at the opposite location to the previous trial - the same pattern of data that the inhibition account predicts (and shows). In contrast to the action co-representation accounts of Ondobaka et al. (2012) and Welsh et al. (2005), Cole et al. (2012) suggested that action representation mechanisms are not necessary for the effect to occur. These authors argued that a body movement, as with any visual 'transient', acts as a cue that shifts an observer's attention to the reached-to location. This shift in attention then induces IOR in the observer at the location of the cue.

In the present experiment, we assessed the action representation and attentional accounts by introducing an additional factor in which lone participants (i.e., with no partner present) were presented with a transient cue that flashed on one side of the display at the location of one of the cards. This cue effectively replaced the arm movement transient that occurs when a co-actor responds in the standard paradigm. In this transient cue condition, participants were asked to perform a reaching response to the location of the cued card. A necessary condition of supporting the attention shift account is that the same pattern of data should be observed when a co-actor's response is replaced by an attention capturing transient i.e., an increase in RT on same target trials relative to different target trials in the absence of a goal-directed movement.

\section{Method}


Stimuli and Apparatus. Stimuli presentation and response recording was programmed using PsychoPy libraries for psychophysics in Python (Peirce, 2007). This experiment was executed from a MacBook Pro computer. All stimuli were presented on a $1920 \mathrm{x}$ 1080 resolution 23” touchscreen that was placed on a table. A pair of Sennheiser headphones delivered response instructions to the co-actor throughout the procedure. The cue was a brief (150 ms) luminance change in which the card was replaced by a uniformly white rectangle $\left(65.0 \mathrm{~cd} / \mathrm{m}^{2}\right)$.

Design and procedure. A 2 × 2 × 2 within-participants design was employed in which location (repeated or opposite), goal (same or different), and cue type (action or luminance) were manipulated. The cue type factor varied whether the confederate coactor responded prior to the participant's response or this was replaced by one of the two cards briefly changing luminance. Thus on action trials, the co-actor made a response to either the higher or lower card on the trial prior to that of the participant. On luminance trials by contrast, a luminance change occurred at the location of either the higher or lower value card. The levels of the cue type and goal factors were blocked in four experimental conditions: 1) action cue, same goal, 2) action cue, different goal, 3) luminance cue, same goal, and 4) luminance cue, different goal. The procedure for the action cue blocks directly replicated that of Ondobaka et al. (2012). In each block, participants completed 168 trials, consisting of 84 same location trials and 84 different location trials. At the beginning of the co-actor cue blocks, participants were told to observe the location of the co-actor's response and to adopt the same card goal during their own response. Each trial of these two blocks began with the display of the fixation screen for $1000 \mathrm{~ms}$. The audio response instruction was delivered to the co-actor and consisted of a wave sound lasting $500 \mathrm{~ms}$ with either the words "higher" or "lower". Following the instruction, the co-actor 
cards would be revealed and he then reached to either the higher or lower card. As soon as contact was made with the touchscreen, the card stimuli would disappear and the co-actor returned their hand to the start button. After a duration of 500ms, the participant's card appeared and remained visible until their subsequent response made contact with the touchscreen. Trials terminated when the participant returned his or her index finger to the start button. For luminance blocks, participants were asked to observe the location of the cue presentation and adopt the same card goal during their own response. These trials began with fixation for $1500 \mathrm{~ms}$, which equated to the time in co-actor trials for the presentation and audio instruction cue. Following fixation, the cards were presented for $1500 \mathrm{~ms}$, which approximated the typical movement times of co-actors. After card stimuli fixation, one of the cards illuminated using a white transient presented for $150 \mathrm{~ms}$. Following the presentation of the cue, the card stimuli disappeared and the participant's trial was initiated as in co-actor trials. Unlike all other experiments reported here, the interval between target presentation and touching of the screen was used as RT.

\section{Results and discussion}

There were $2.9 \%$ of responses defined as outliers and omitted from further analysis. Figure 11 shows mean RTs. An ANOVA with target location, goal, and cue type as within-participants factors revealed significant effects of target location, $\mathrm{F}(1$, $15)=12.3, \mathrm{p}=.003, \eta_{p}^{2}=.45, B F_{10}=.40$, and cue type, $\mathrm{F}(1,15)=21.0, \mathrm{p}<.001$, $\eta_{p}^{2}=.58, B F_{10}=29510.60$, but no significant effect of goal, $\mathrm{F}(1,15)=1.8, \mathrm{p}>.19$, $\eta_{p}^{2}=.1, B F_{10}=.89$. Analysis of the interactions between goal and cue type, $\mathrm{F}(1,15)$ $=.32, \mathrm{p}=.58, \eta_{p}^{2}=.02, B F_{10}=13384.53$, target location and cue type, $\mathrm{F}(1,15)=$ 
1.42, $\mathrm{p}=.25, \eta_{p}^{2}=.086, B F_{10}=3594.44$, and goal and target location, $\mathrm{F}(1,15)=$ 1.64, $\mathrm{p}=.35, \eta_{p}^{2}=.041, B F_{10}=.09$, showed no significant effects. The three-way interaction was also not significant, $\mathrm{F}(1,15)=.06, \mathrm{p}=.77, \eta_{p}^{2}=.01 B F_{10}=160.42$. Bayesian analysis compared the interaction model for goal and target location as well as that for cue type and location, to a model specifying a main effect of target location and cue type only. This revealed preference for the main effects model over the interaction model between goal and location, $B F_{10}=21651.61$, and the interaction model between cue type and location, $B F_{10}=29059.90$. These results correspond to decisive evidence in favour the alternative hypothesis that there was a main effect of both cue type and target location over an interaction between location and goal. The Bayesian results also correspond to decisive evidence in favour of an alternative hypothesis of these main effects over an interaction between cue type and target location.

With respect to the aims of the present experiment, the most important findings concern evidence in favour of an effect of target location, which was independent of goal or cue type. Thus, we have found that the basic reaching phenomenon was not modulated according to the goals of co-actors and thus supports the findings of Experiments 2-6 and of Cole et al. (2012). That is, the effect does not appear to be goal-based. We have also found the target location effect to be the same irrespective of whether a co-actor made a reaching response or his action was replaced by a transient cue in his absence. Although this finding does not necessarily refute the action co-representation account, it does clearly show that IOR can be elicited by visual transients occurring at a given location - a conclusion consistent with an attention-based account of the basic effect. 


\section{Cross-experiment meta-analysis and replication}

Although none of the present seven experiments, nor the three reported by Cole et al. (2012), support the goals-based theory of movement, it is possible that such a phenomenon does exist but that the effect is small. We therefore performed a metaanalysis combining the data from the seven present experiments and the three of Cole et al. (2012). For all 10 data sets, we analysed both levels of the target location factor (repeated, opposite), both levels of the goals factor (same, different), and all 10 levels of the experiment factor, in a 2 × 2 x 10 mixed ANOVA design. The target location and goals factors were repeated measures whilst the experiment factor was independent. Other factors specific to individual experiments (e.g., task relevancy, present Experiment 1) were collapsed within the factors stated above. Furthermore, we did not include the transient conditions of the present Experiment 7 in which no partner was present. Analysis of this much larger data set $(\mathrm{N}=162)$ revealed main effects of target location, $\mathrm{F}(1,152)=64.5, \mathrm{p}<.0001, \eta_{p}^{2}=.3, B F_{10}=5.81$, and experiment, $\mathrm{F}(9,152)=92.1, \mathrm{p}<.0001, \eta_{p}^{2}=.85, B F_{10}=3.00+\mathrm{e} 53$, yet no effect of goals, $\mathrm{F}(1,152)=.04, \mathrm{p}>.85, \eta_{p}^{2}<.001, B F_{10}=.09$. There was no significant target location $\mathrm{x}$ goals interaction, $\mathrm{F}(1,152)=.77, \mathrm{p}>.39, \eta_{p}^{2}<.005, B F_{10}=.07$. The target location $\mathrm{x}$ experiment, $\mathrm{F}(9,152)=1.66, \mathrm{p}=.10, \eta_{p}^{2}=.090, B F_{10}=4.03 \mathrm{e}+51$, goals x experiment, $\mathrm{F}(9,152)=.62, \mathrm{p}=.78, \eta_{p}^{2}=.035, B F_{10}=4.47 \mathrm{e}+51$, and threeway interactions were all non-significant $\mathrm{F}(9,152)=.60, \mathrm{p}=.79, \eta_{p}^{2}=.03, B F_{10}=$ 6.97e+46. Bayesian analysis compared the model for an interaction between goal and target location to the additive model for target location and experiment only. This revealed preference for the additive model, $B F_{10}=9.94$, corresponding to positive 
evidence in favour of the alternative hypothesis that there was an additive effect of experiment and target location over an interaction between goal and target location.

In addition to the meta-analysis and Bayesian analyses described above, we also replicated one of our experiments using $\mathrm{N}=46$. This sample size was shown in an a priori power analysis to be able to detect a medium-to-large effect size (Cohen's $d \geq$ 0.5) with an $80 \%$ probability. Experiment 5 was chosen for replication because of its clearest operational definition of a task goal, i.e., cooperate (or not) with a co-actor in completing a line drawing. The results can be seen in Table 1 . The ANOVA ${ }^{3}$ revealed a significant main effect of target location, $\mathrm{F}(1,44)=21.5, \mathrm{p}<.001, \eta_{p}^{2}=.33, B F_{10}$ $=7.59$, but no significant main effect of goal, $\mathrm{F}(1,44)=3.40, \mathrm{p}>.07, \eta_{p}^{2}=.07, B F_{10}$ $=4.93$. Importantly, the interaction was not significant, $\mathrm{F}(1,44)=.48, \mathrm{p}=.49, \eta_{p}^{2}=$ $.01 B_{10}=11.03$ and Bayes factors revealed moderate evidence in favour of an additive main effects model compared to an interaction model, $B F_{10}=4.26$, corroborating with a large sample size our earlier results. The coding of goals, if it occurs, does not influence the planning of a repeated action.

\section{General Discussion}

An abundance of work has shown that the observation of goal-directed behaviour can influence the actions performed by an observer. This is most clearly evident when two people cooperate on a joint action task such as moving a piece of furniture. Furthermore, evidence from a number of studies shows that observers are more likely to copy an action outcome than copy the means by which the outcome is achieved (e.g., Bekkering, et al. 2000). Moreover, many influential theories of social facilitation emphasize the primacy of goals over action kinematics (e.g., Carpenter, Akhtar, \& Tomasello, 1998; Gattis, Bekkering, \& Wohlschläger, 2002) and one of the 
mechanisms said to subserve action understanding, i.e., the mirror neuron system (di Pellegrino, Fadiga, Gallese, \& Rizzolatti, 1992), is thought to represent common goals rather than common body movement (e.g., Rizzolatti, Fabbri-Destro \& Cattaneo, 2008).

However, one question of interest is whether the underlying goals are encoded when an observed action elicits an equivalent internal representation in the observer; that is, during direct matching of a specific movement. Although Wohlschläger and Bekkering (2002) did find an influence of goals on movements, whilst Chiavarino et al.'s (2013) found a counterintuitive effect of goal compatibility; i.e., a larger behavioural effect when participants' goals differed from those of a model. Furthermore, employing the Welsh et al. (2005) arm reaching paradigm, Ondobaka et al. (2012; 2013) and Cole et al. (2012) reported contrasting results: whilst Ondobaka found a goals-based effect of action Cole et al. did not. The current study therefore set out to examine the conditions under which specific movements (e.g., reaching to a location) are influenced by goals and the conditions under which they are not. To that end, we examined goals and movements in the context of task relevancy, end-point action versus selection, objects and locations, and top-down versus bottom-up determination of response position. Thus, by isolating the main differences between the experiments of Cole et al. and Ondobaka et al., we assessed an action observation phenomenon with reference to a number of theoretical processes. However, in seven experiments, including two direct replications of Ondobaka et al. (2012; 2013), a meta-analysis and a separate large-sample replication of one of our own experiments, we have found no evidence that movements are influenced by the goals of co-actors. Neither the most conservative form of the goal-based theory, that is, spontaneous recognition of goals, nor the most liberal were supported. Although Experiment 1 did 
show that the reaching phenomenon was influenced by goals, this was in the opposite direction to what might be expected, and indeed the direction reported by Ondobaka et al. (2012); the effect was smaller when participants adopted the same goal. Furthermore, although no effect of goals on the present action observation effect was observed in Experiment 2, the critical (target location by goals) interaction did reveal a small but non-significant effect, $\mathrm{p}=.11, \eta_{p}^{2}=.17, B F_{10}=5.86$. This experiment therefore, for whatever reason, may be the most sensitive to any goals-based effect, if it does occur. Any future work examining goals and action using the present paradigm may want to develop this particular experiment.

At which level of representation do goals influence actions?

This question relates to one of the main issues facing any research aimed at assessing goals, and one highlighted by Cole et al. (2012) and Ondobaka et al. (2012); namely how should a particular goal be specified. Since the early ideas of James (1890) and McDougall (1908), goals have meant different things to different researchers. A fundamental distinction however relates to the difference between proximal and distal goals (e.g., Bandura, 1989), notions commonly used in the philosophy of action and the social psychology of goals. In keeping with Bekkering et al. (2000), Ondobaka et al. referred to the fact that intentions can be specified at multiple levels (see also Uithol, Burnston, \& Haselager, 2014; Pacherie, 2008) and in particular made the distinction between movement intention (e.g., picking up a sponge with the aim of rotating it) and action intention (e.g., cleaning dirty dishes with the sponge). Similarly, Cole et al., made reference to the fact that a movement goal can be separated into subcomponents such as directed reach, object grasp, and return movement, a notion that is supported by patient work in which one or more 
components can be separately affected (e.g., Sirigu et al., 1995). Thus, even the proximal intention of a simple action has a number of subgoals associated with it. Furthermore, as much as these components are subserved by distinct anatomical areas, goals, as noted, differ according to how experimenters conceive them. This point was made by the behaviourists who were concerned with goals in the context of motivational need theories (Atkinson, 1964; Lewin, 1926; McClelland, 1951; Murray, 1938). As Gollwitzer and Moskowitz (1996) pointed out in an extensive review of how goals affect cognition and action, the behaviourists did not examine "how a selfset goal affects behavior. For the behaviorist, a goal is just an incentive that is chosen by the investigator as a reference point for describing observed behavior”. Thus, Skinner (1953) described goals as simply an 'abbreviation' for describing (operant) behaviour. One does not however have to reject the cognitive revolution in order to accept that the conception of goal in the context of joint action is experimenterdependent. For instance, in Ondobaka et al. (2013) participants were required to respond as rapidly as possible whilst withholding a response on 'catch' trials. By contrast, their confederate co-actor was not presented with these no-go trials and did not therefore have to be concerned with inhibiting a response. The goals of each were thus always different. At the same time, co-actors were always required to attend to the numbers on the cards. Thus, their goals were also always the same. Despite the different ways in which goals can be conceived, we contend that in terms of goals and actions it is most useful to distinguish between movement intention and action intention, as Ondobaka et al. (2012) did. The evidence from previous work appears to show that goals are represented in the general sense in which action can be conceived (e.g., cleaning dirty dishes with a sponge). The present work however suggests that 
goals are not predominantly represented with respect to specific movements (e.g., picking up a sponge and rotating it).

The issue of goal definition may also help explain the contrasting results found by the present experiments and those of past studies showing that goals can modulate specific movements (e.g., Bouquet, et al. 2010; Liepelt et al., 2008; Wohlschläger \& Bekkering, 2002). Previous studies have tended to manipulate inferred goals based on some manipulation of the stimuli. For instance, Bouquet et al. (2010) presented participants with a video showing an actor perform arm reaching movements. In the 'non-goal-directed' conditions the actor simply reached out into empty space. To generate the 'goal-directed' conditions, the same video sequence was shown with the exception that a large circular red dot was superimposed onto the film at the location of the actor's end-point reach. Because participants were not explicitly told that the actor's goal was to reach to a red dot, the experimental set-up required participants to infer that the dots were part of the actor's goal. Of course, this does not negate the fact that Bouquet et al. did observe a difference in participant reaching behaviour when the dots were present or not. However, the fact that the dots were clearly not present or visible to the actor means that it is difficult to interpret the result in terms of goal state attribution. By contrast, in the present experiments goals did not need to be implicitly inferred; participants were always told “your goal is the same” or "your goal will be different on this block".

What causes the same-location slowing effect?

Although the primary aim of the present work was to examine the issue of goals in movement planning, Experiment 7 has provided evidence pertaining to the possible cause of the basic effect reported by Welsh et al. (2005). As described in the Introduction, Welsh and colleagues placed the effect within the context of the mirror 
neuron system and perceptual-motor models (e.g., Hommel, 2009) that suggest observed actions are incorporated within one’s own action planning. Similarly, Ondobaka et al. (2012) also argued that the effect is due to the representation of actions. Specifically, the initiation of a movement that is congruent (i.e., imitates) with one just observed was said to be facilitated. In contrast to these action representation theories, Cole et al. suggested that there is nothing particularly special about action per se because any motion transient can induce IOR (see Abrams \& Christ, 2003). Furthermore, this explanation is not refuted by experiments showing that the reaching effect still occurs when peripheral motion transients are obscured either by opaque goggles (Welsh et al., 2007) or physical barriers (Skarratt et al., 2010). Here, the cue becomes the initial portion of the movement; what is commonly referred to as a 'central' cue in the attentional cueing literature (e.g., Cole, Smith, \& Atkinson, 2015). Additionally, the phenomenon may be part of a range of effects collectively referred to as social attention. Common to all of these behaviours is the disposition to direct attention to locations of interest in the environment as a result of social cues. These cues can include gaze, gesture and goal-directed action and can induce shifts of attention, as well as IOR (Birmingham \& Kingstone, 2009; Gervais, Reed, Beall, \& Roberts, 2010). Indeed, there is some evidence that such social cues operate via channels that are relatively independent from motion cues, at least with respect to gaze cues and head motion (Böckler, van der Wel, \& Welsh, 2014). Although Welsh and colleagues include attentional orienting as part of their account (i.e., IOR), action representation is still central to their explanation. By contrast, the Cole et al. account predicts that any luminance cue, including a brief luminance change at the location of a card, should induce the effect. This is precisely what was found in Experiment 7: Irrespective of whether a co-actor responded, or this action 
was replaced by a luminance cue, a same/different target RT effect occurred. The hypothesis that the effect is due to the capture of attention by motion cues (e.g., a moving arm), and the subsequent IOR, supports recent work arguing that the phenomenon is primarily attentional in origin (e.g., Atkinson et al., 2014; Doneva \& Cole, 2014). However, as we noted in Experiment 7, observing 'social IOR'-like data when a co-actor's response is replaced by a luminance cue does not on its own refute the action representation account. This finding does, however, suggest that the shift of attention is likely a necessary condition for the effect to emerge.

\section{Attention in joint action}

The results from Experiment 7 also concern a more general issue regarding attentional cueing in joint action. The vast majority of action observation work, including review articles (e.g., Galantucci \& Sebanz, 2009), make no reference to attention (e.g., Braun, Ortega, \& Wolpert, 2011; Paulus \& Moore, 2007; Vesper, van der Wel, Knoblich, \& Sebanz, 2007; Atmaca, Sebanz, \& Knoblich, 2007). When attention is mentioned it is never acknowledged that attentional cueing may play a significant role in an effect. Indeed, the potential confound of action as an attentional cue has been shown in the most common joint action paradigm. As with the present paradigm, the 'joint Simon effect' shows that the presence of another acting partner modulates one's own responses and has similarly been explained with reference to the representation of observed and executed action. However, similar to the present Experiment 7, Dolk, et al., (2013) showed that the effect can be induced by any sufficiently salient stimulus that attracts attention (and induces a 'referential code'; Hommel, 1993) to where the co-actor would normally respond, for example, a clock that emits an audible clicking sound. Thus, a phenomenon that was believed to be 
caused by the integration of another person's action into one's own action planning appears to be due to attentional orienting.

In sum, previous work has shown that the goals of other people's actions can influence the general actions of an observer. In the present work, we have examined the circumstances under which specific actions, influenced by the observation of similar movements, are modulated by the goals of those observed actions. We found that although a reaching action is modulated by the observation of the same action, this was not influenced by the goals of the action. This set of findings indicates that the mechanisms that code for action kinematics can have a primary influence on action planning; a conclusion that stands in contrast to the primacy of goal coding.

\section{References}

Abrams, R. A. \& Christ, S. E. (2003). Motion onset captures attention. Psychological Science, 14, 427-432.

Atkinson, J. W. (1964). An introduction to motivation. Princeton, NJ: Van Nostrand.

Atkinson, M., Skarratt, P.A., Simpson, A., \& Cole, G. G (2014). Is social IOR due to action co-representation? Acta Psychologica, 150, 85-93.

Atmaca, S., Sebanz, N., \& Knoblich, G. (2011). The joint flanker effect: Sharing tasks with real and imagined coactors. Experimental Brain Research, 211, 371385.

Bandura, A. (1989). Self-regulation of motivation and action through internal standards and goal systems. In L. A. Pervln (Ed.), Goal concepts in personality and social psychology (pp. 19-85). Hillsdale, NJ: Erlbaum.

Bekkering, H., Wohlschläger, A., \& Gattis, G. (2000). Imitation of gestures in children is goal-directed. Quarterly Journal of Experimental Psychology: 
Human Experimental Psychology, 53(A), 153-164.

Birmingham, E. \& Kingstone, A. (2009). Human Social Attention. Annals of the New York Academy of Sciences, 1156: 118-140.

Böckler, A., van der Wel, R.P., \& Welsh, T.N. (2014). Catching eyes: Effects of social and non-social cues on attention capture. Psychological Science, 25, 720-727.

Bouquet, C. A., Shipley, T. F., Capa, R. L., \& Marshall, P. J. (2011). Motor contagion: Goal-directed actions are more contagious than non-goal-directed actions. Experimental Psychology, 58, 71-78.

Braun, D. A., Ortega, P.A., Wolpert, D. M. (2011). Motor coordination: when two have to act as one Experimental Brain Research 211, 631-641.

Brass, M., Bekkering, H., Wohlschläger, A., \& Prinz, W. (2000).

Compatibility between observed and executed finger movements: comparing symbolic, spatial, and imitative cues. Brain and Cognition, 44, 124-143.

Carpenter, M., Akhtar, N., \& Tomasello, M. (1998). Fourteen- through

18-month-old infants differentially imitate intentional and accidental actions. Infant Behavior and Development, 21, 315-330.

Chiavarino, C., Bugiani, S., Grandi, E., \& Colle, L. (2013). Is automatic imitation based on goal coding or movement coding? Experimental Psychology, 60, 213225.

Cohen, J. (1962). Statistical power of abnormal-social psychological research: A review. Journal of Abnormal and Social Psychology, 65, 145-153.

Cole, G. G., Skarratt, P.A., \& Billing R-C. (2012). Do action goals mediate social inhibition of return? Psychological Research, 76, 736-746.

Cole, G. G., Smith, D, \& Atkinson, M. (2015). Mental state attribution and the gaze 
cueing effect. Attention, Perception \& Psychophysics, 77, 1105-1115.

Cole, G. G., Wright, D., Doneva, S. \& Skarratt, P. A. (2015). When your decisions are not (quite) your own: Action observation influences free choices. PLoS ONE 10(5): e0127766.

Crundall, D., Cole, G. G., \& Galpin, A. (2007). Object-based attention is mediated by collinearity of targets. Quarterly Journal of Experimental Psychology, 60, 137-153

Csibra, G., Gergely, G., Bıro, S., Koos, O., \& Brockbank, M. (1999). Goal attribution without agency cues: The perception of pure reason in infancy. Cognition, 72, 237-267.

Dolk, T., Hommel, B., Prinz, W., \& Liepelt, R. (2013). The (not so) social simon effect: A referential coding account. Journal of Experimental Psychology: Human Perception and Performance, 39, 1248-1260.

Doneva, S., \& Cole, G. G. (2014). The role of attention in a joint-action effect. PLOS One, 9, 3, e91336.

Duncan, J. (1984). Selective attention and the organization of visual information. Journal of Experimental Psychology: General, 113, 501-517.

di Pellegrino, G., Fadiga, L., Gallese, V., \& Rizzolatti, G. (1992). Understanding motor events: A neurophysiological study. Experimental Brain Research, 91, 176-180.

Egly, R., Driver, J., \& Rafal, R. D. (1994). Shifting visual attention between objects and locations: Evidence from normal and parietal lesion subjects. Journal of Experimental Psychology: General, 123, 161-177.

Folk, C. L., Remington, R. W., \& Johnston, J. C. (1992). Involuntary covert orienting is contingent on attentional control settings. Journal of Experimental 
Psychology: Human Perception and Performance, 18, 1030-1044.

Galantucci, B., \& Sebanz, N. (2009). Joint action: Current perspectives. Topics in Cognitive Science, 1, 255-259.

Gattis, M., Bekkering, H., \& Wohlschläger, A. (2002). Goal-directed imitation.

In A. N. Meltzoff \& W. Prinz (Eds.), The imitative mind (pp. 183-205).

Cambridge, England: Cambridge University Press.

Gervais, W. M., Reed, C. L., Beall, P. M., Roberts R. J. Implied body action directs spatial attention. Attention, Perception, \& Psychophysics, 72, 1437-1443.

Gollwitzer, P. M., \& Moskowitz, G. B. (1996). Goal effects on action and cognition. In E.T. Higgins \& A. Kruglanski (Eds.), Social Psychology: Handbook of basic principles, 361399, New York: Guildford.

Hassin, R. R., Aarts, H., \& Ferguson, M. J. (2005). Automatic Goal Inferences. Journal of Experimental Social Psychology, 41, 129-140.

Hayes, S. J., Hansen, S., \& Elliot, D. (2010). Between-person effects on attention and action: Joe and Fred revisited. Psychological Research, 74, 302-312.

Heider, F., \& Simmel, M., (1944). An experimental study of apparent behavior. American Journal of Psychology 57, 243-259.

Hommel, B. (1993). The role of attention for the Simon effect. Psychological Research, 55, 208-222.

Hommel, B. (2009). Action control according to TEC (theory of event coding) Psychological Research, 73, 512-526.

Hudson, M., Nicholson, T., Ellis, R., \& Bach, P. (2016). I see what you say: Prior knowledge of other's goals automatically biases the perception of their actions, Cognition, 146, 245-250.

Iacoboni, M., Woods, R. P., Brass, M., Bekkering, H., Mazziotta, J. C., \& 
Rizzolatti, G. (1999). Cortical mechanisms of human imitation. Science, 286, 2526-2528.

Itti, L., \& Koch, C. (2000). A saliency-based search mechanism for overt and covert shifts of visual attention. Vis Res, 40, 1489-1506.

James, W. (1890). Principles of psychology. New York: Holt.

Jeannerod,M. (1999). The 25th Bartlett Lecture: to act or not to act: perspectives on the representation of actions. The Quarterly Journal of Experimental Psychology, 52, 1-29.

Jordan, H. \& S. P. Tipper. (1999). Spread of inhibition across an object's surface. British Journal of Psychology, 90: 495-507.

Kilner, J., Paulignan, Y., \& Blakemore, S. (2003). An interference effect of observed biological movement on action. Current Biology, 13 (6), 522-525.

Knoblich, G., \& Sebanz, N. (2006). The social nature of perception and action. Current Directions in Psychological Science, 15, 99-104.

Koole, S. L., \& Lakens, D. (2012). Rewarding replications: A sure and simple way to improve psychological science. Psychological Science, 7, 608-614.

Lewin, K. (1926). Vorsatz, wille und bedurfnis. Psychologische Forschung, 7, 330-385.

Liepelt, R., von Cramon, D. Y., \& Brass, M. (2008). What is matched in direct matching? Intention attribution modulates motor priming. Journal of Experimental Psychology: Human Perception and Performance, 34, 578-591.

Longo, M. R., Kosobud, A., \& Bertenthal, B. I. (2008). Automatic imitation of biomechanically possible and impossible actions: Effects of priming movements versus goals. Journal of Experimental Psychology: Human Perception and Performance, 34, 489-501.

Lyons, J., Weeks, D. J., \& Elliott, D. (2013). The Gambler's Fallacy: A Basic 
Inhibitory Process? Frontiers in Psychology, 4, 72.

Love J., Selker R., Marsman M., Jamil T., Verhagen A. J., Ly A., et al. (2015). JASP (Version 0.6.6) [Computer software].

Milner, A. D., \& Goodale, M. A. (1995). The visual brain in action. Oxford: Oxford Press.

McClelland, D. C. (1951). Personality. New York: William Sloane Associates.

McDougall, W. (1908). Social psychology. London: Methuen.

Murray, H. A. (1938). Explorations in personality. New York: Oxford University Press.

Morey, R. D., \& Rouder, J. N. (2011). Bayes factor approaches for testing interval null hypotheses. Psychological Methods, 16, 406-419.

Ondobaka, S., de Lange, F. P., Newman-Norlund, R. D., Wiemers, M., \& Bekkering, H. (2012). Interplay between action and movement intentions during social interaction. Psychological Science, 23, 30-35.

Ondobaka, S., Newman-Norlund, R,D., de Lange F.P., Bekkering, H. (2013). Action recognition depends on observer's level of action control and social personality traits. PLoS ONE, 8, 11, e81392.

Paternotte, C. (2014). Constraints on joint action. In M. Gallotti, \& J. Michael. Perspectives on Social Ontology and Social Cognition. PP, 103-125. Springer: Dordrecht.

Paulus, M., \& Moore, C. (2011). Whom to ask for help? Children’s developing understanding of other people’s action capabilities. Experimental Brain Research, 211, 593-600.

Peirce, J. W. (2007). PsychoPy: Psychophysics software in Python. Journal of Neuroscience Methods, 162, 8-13. 
Peters, J., Suchan, B., Zhang,Y., \& Daum, I. (2005). Visuo-verbal interactions in working memory - evidence from event-related potentials. Cognitive Brain Research, 25, 406-515.

Piaget, J. (1929). The child's conception of the natural world. London: Routledge and Kegan Paul.

Posner, M. I., \& Cohen, Y. (1984). Components of visual orienting. In H. Bouma \& D. G. Bouwhuis (Eds.), Attention and Performance X (pp. 531-556). Hillsdale, NJ: Erlbaum.

Raftery, A. E. (1995). Bayesian model selection in social research. Sociological Methodology, 25, 111-163.

Rieger, M., \& Gauggel, S. (1999). Inhibitory after-effects in the stop-signal paradigm. British Journal of Psychology, 90, 509-518.

Prinz, W. (1997). Perception and action planning. European Journal of Cognitive Psychology, 9, 129-154.

Rizzolatti, G., Fabbri-Destro, M., \& Cattaneo, L. (2008). Mirror neurons and their clinical relevance, Nature Clinical Practice Neurology, 5, 24-34.

Schmidt, S. (2009). Shall we really do it again? The powerful concept of replication is neglected in the social sciences. Review of General Psychology, 13, 90-100.

Sebanz, N. \& Knoblich, G. (2009). Prediction in joint action: What, when, and where. Topics in Cognitive Science, 1, 353-367.

Sebanz, N., Knoblich, G., \& Prinz, W. (2003). Representing others’ actions: just like one’s own? Cognition, 88, 11-21.

Sebanz, N., Knoblich, G., \& Prinz, W. (2005). How two share a task. Journal of Experimental Psychology: Human Perception and Performance, 31, 12341246. 
Simon, J. R. (1969). Reactions toward the source of stimulation. Journal of Experimental Psychology, 81, 174-176.

Sirigu, A., Cohen, L., Duhamel, J.R., Pillon, B., Dubois, B. \& Agid, Y. (1995). A selective impairment of hand posture for object utilisation in apraxia, Cortex, 31, 41-55.

Skarratt, P. A., Cole, G. G., Kingstone, A. (2010). Social inhibition of return. Acta Psychologica, 134, 48-54.

Skinner, B. F. (1953). Science and human behavior. New York

Sun, H. M., \& Thomas, L. E. (2013). Biased attention near another's hand following joint action. Frontiers in Psychology, 4, 443.

Tipper, S. P., Jordan, H., \& Weaver, B. (1999). Scene-based and object-centered inhibition of return: Evidence for dual orienting mechanisms. Attention, Perception, \& Psychophysics, 61, 50-60.

Tsang, E., \& Kwan, K. (1999). Replication and theory development in organizational science: A critical realist perspective. Academy of Management Review, 24, 759780.

Uithol, S., Burnston, D. C., \& Haselager, P. (2014). Why we may not find intentions in the brain. Neuropsychologia, 56, 129-139.

Van der Cruyssen, L.,Van Duynslaeger., M., Cortoos, A., and Van Overwalle, F.(2009). ERP time course and Brain areas of spontaneous and Intentional goa linferences. Social Neuroscience. 4, 165-184.

Vesper, C., van der Wel, R.P.R.D., \& Sebanz, N., \& Knoblich, G. (2011). Making oneself predictable: Reduced temporal variability facilitates joint action coordination. Experimental Brain Research, 211, 517-530. 
Wagenmakers, E. J. (2007). A practical solution to the pervasive problems ofp values. Psychonomic bulletin \& review, 14(5), 779-804.

Welsh, T.N., Manzone, J., \& McDougall, L.M. (2014). Knowledge of response location alone is not sufficient to generate social inhibition of return. Acta Psychologica, 153, 153-159.

Welsh, T. N., Elliot, D., Anson, J. G., Dhillon, V., Weeks, D. J., Lyons, J. L., \& Chua, R. (2005). Does Joe influence Fred's actions? Inhibition of return across different nervous systems. Neuroscience Letters, 385, 99-104.

Welsh, T. N., Lyons, J., Weeks, D. J., Anson, J. G., Chua, R., Mendoza, J., \& Elliot, D. (2007). Within- and between-person inhibition of return: Observation is as good as performance. Psychonomic Bulletin \& Review, 14, 960-956.

Welsh, T. N., McDougall, L. M., \& Weeks D. J. (2009). The performance and observation of action shape future behaviour. Brain and Cognition 71, 64-71.

Welsh, T. N., Ray, M. C., Weeks, D. J., Dewey, D., \& Elliott, D. (2009). Does Joe influence Fred's action? Not if Fred has autism spectrum disorder. Brain Research, 1248, 141-148.

Wohlschläger, A., \& Bekkering, H. (2002). Is human imitation based on a mirrorneurone system? Some behavioural evidence. Experimental Brain Research, 143, 335-341. 


\section{Footnotes}

1) It is not yet known why the arm reaching phenomenon occurs. Welsh et al. (2005) and Ondobaka et al. (2012) both argued that action representation mechanisms play a role in the basic effect. However, much like Dolk, Hommel, Prinz and Liepelt, (2013) with respect to the 'joint Simon effect', Cole et al. (2012) have raised the possibility that the effect is associated with the attentional shifts generated by observing an action. It is also possible that both motor and attentional mechanisms contribute to the effect with different degrees of contribution of each being dependent on the conditions of the task. See the present Experiment 7 and General Discussion.

2) As pointed out by a number of authors (e.g., Schmidt, 2009; Koole \& Lakens, 2012), a direct replication is a study in which 'all the relevant aspects' (Schmidt, 2009) of the original study are repeated. This contrasts a conceptual replication in which a hypothesis is tested using a different design (Tsang \& Kwan, 1999). Koole and Lakens, (2012) also noted that 'a given researcher is inevitably prone to the idiosyncrasies in procedural details or how she or he treats participants (to name two examples)'. Furthermore, although over 50 years have passed since the seminal paper of Cohen (1962), experimental psychology has only recently become concerned with issues regarding effect sizes, $p$-values, replication, and their associates. Moreover, whilst asking 'Why do psychology journals systematically refuse to publish replications?', Schmidt (2009) notes that 'replications are likely to become associated with controversy. This is unproductive and unjustified'.

3) The analysis was conducted on $\mathrm{N}=45$ due to the omission of one participant for making a large number ( $>20 \%)$ of anticipatory errors. 


\section{Figure captions}

Figure 1. Schematic representation of the standard action observation paradigm reported by Welsh et al. (2005) and Skarratt et al. (2010). Results typically show that co-actors are slower to initiate a reaching action to the location that their partner just reached to, or conversely, they are quicker to reach to the opposite location.

Figure 2. Results from Experiment 1.

Figure 3. The stimuli employed in Experiment 2.

Figure 4. Results from Experiment 2.

Figure 5. The stimuli employed in Experiment 3. The upper panel shows the 'separate' condition, the lower panel shows the 'grouped’ condition.

Figure 6. Results from Experiment 3.

Figure 7. Results from Experiment 4.

Figure 8. The experimental set-up, including the dot-to-dot house and pencil, employed in Experiment 5. The figure also shows the cue. Note that the house comprised 103 dots rather than the 66 shown on the figure.

Figure 9. Results from Experiment 5.

Figure 10. Results from Experiment 6.

Figure 11. Results from Experiment 7.

\section{Table caption}

Mean RTs (and standard deviations; both in ms) from the replication of Experiment 5, comparing actions as a function of response location and goal compatibility. The magnitude of same-location slowing is reported in the right column, and does not differ according to goal compatibility. 


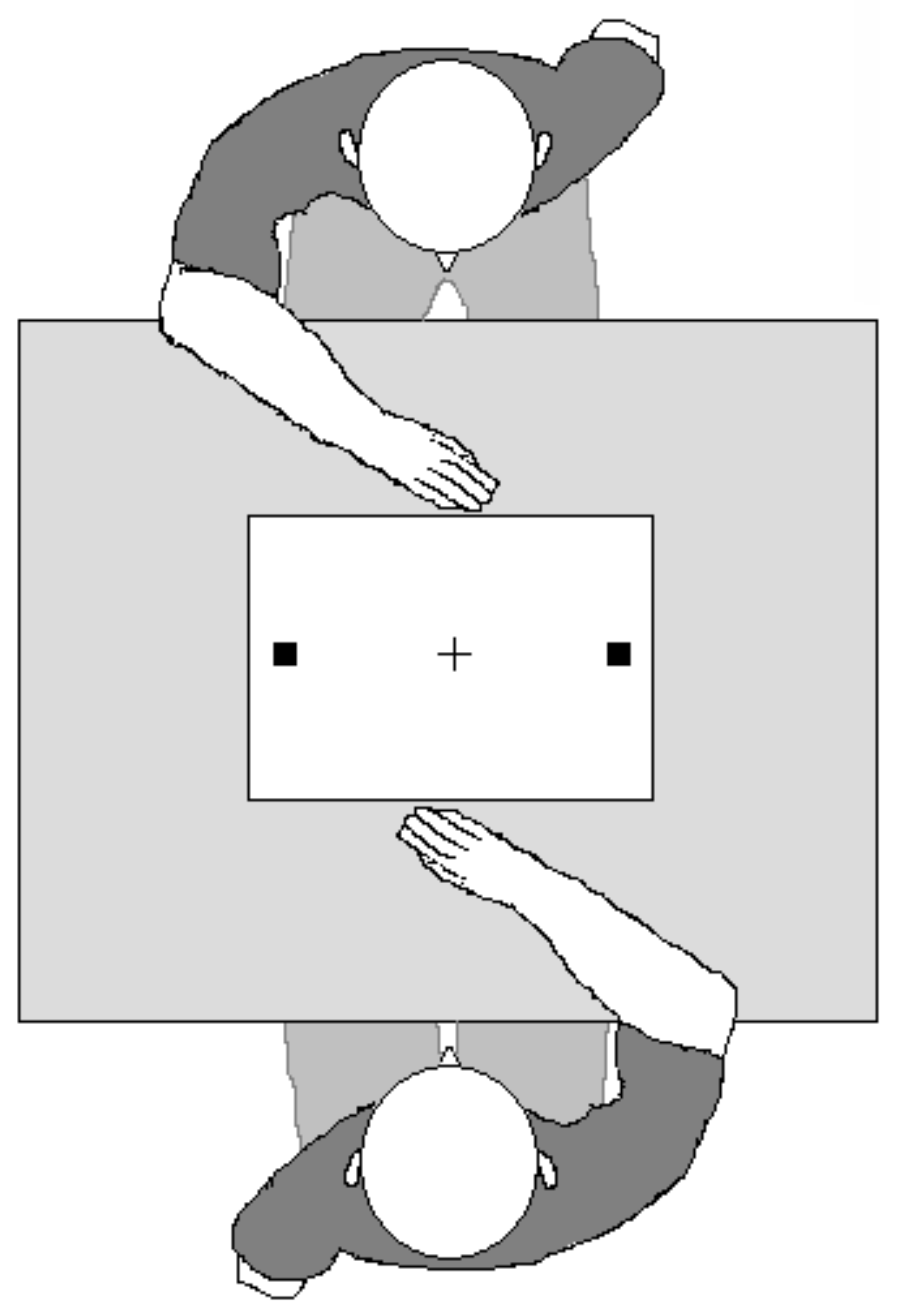

Figure 1 


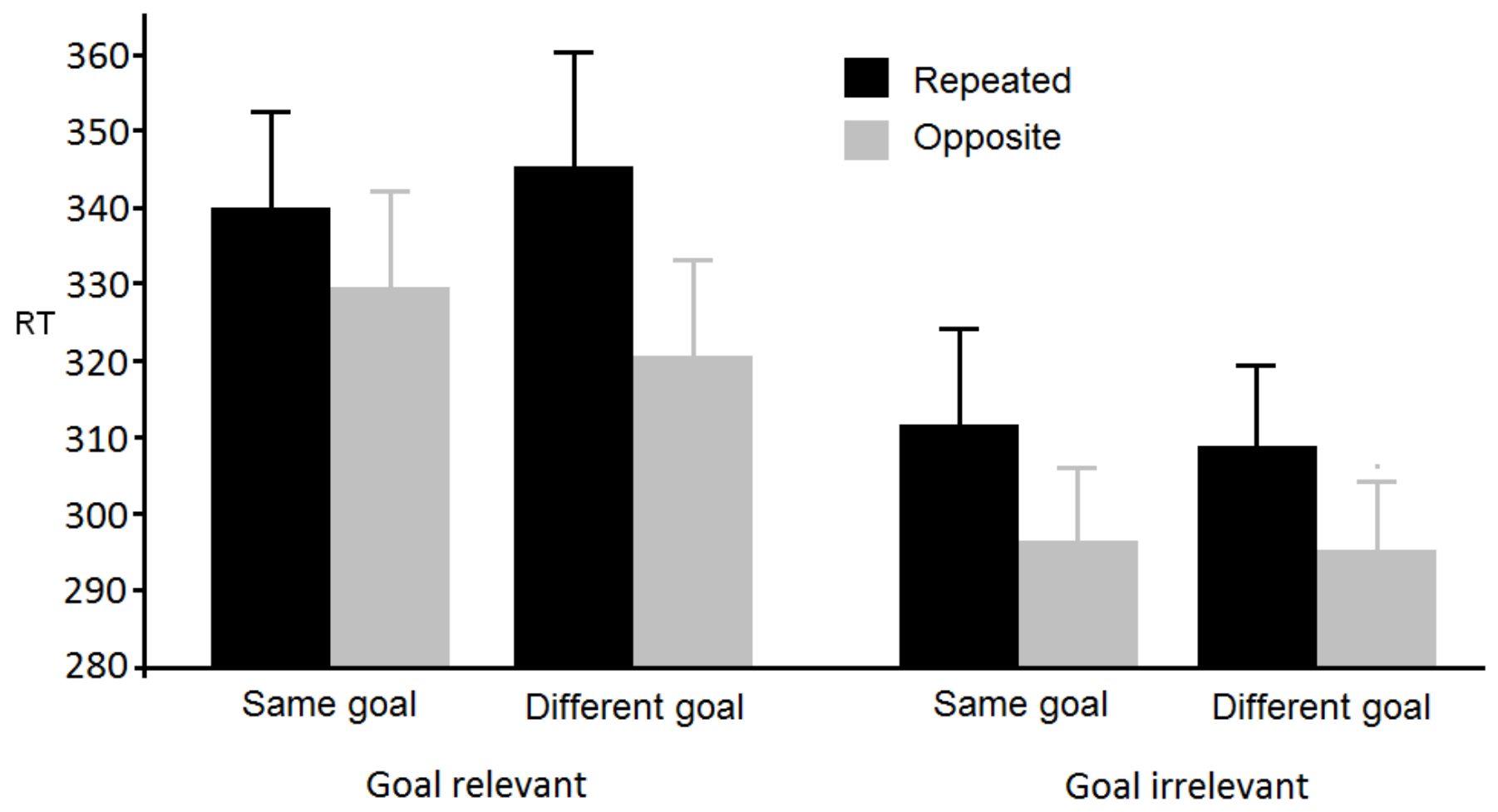

Figure 2 


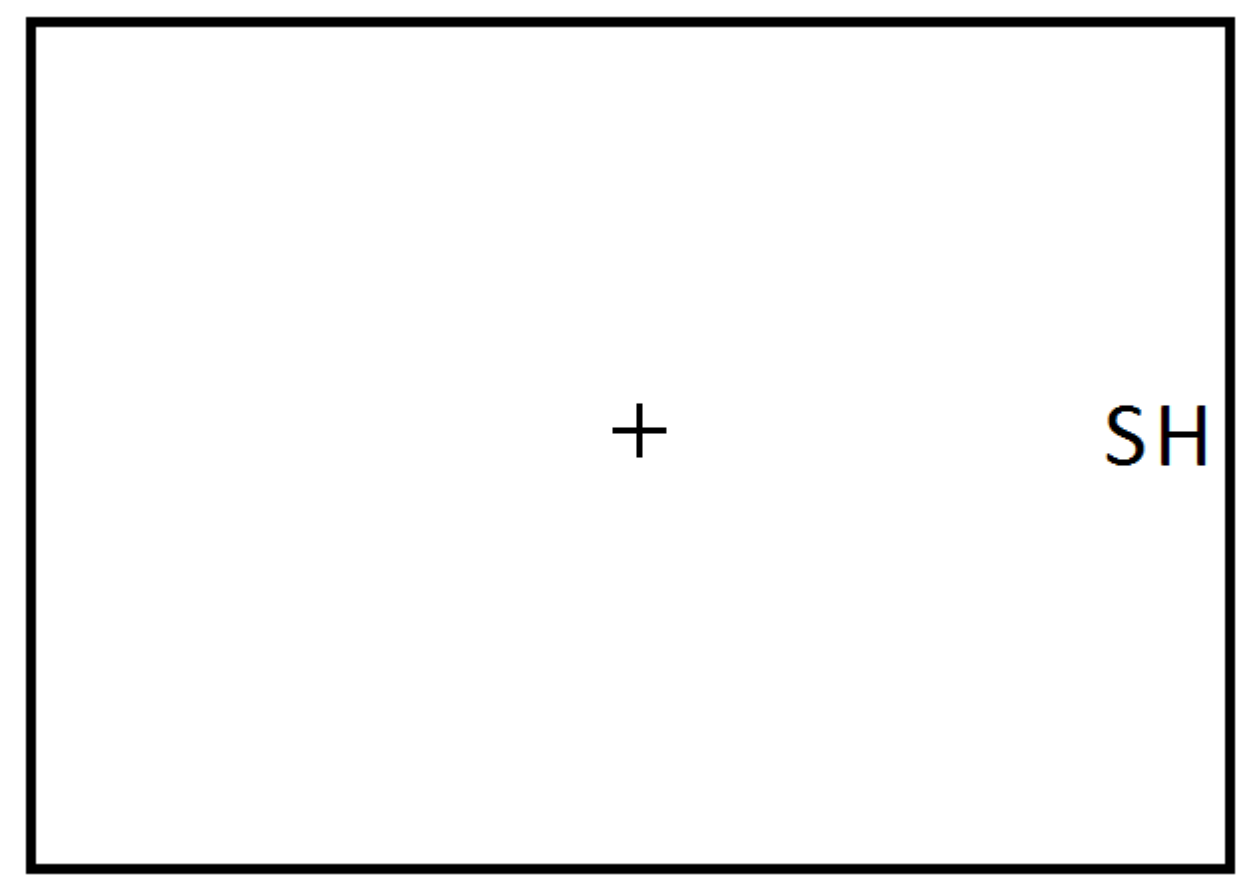

Figure 3 


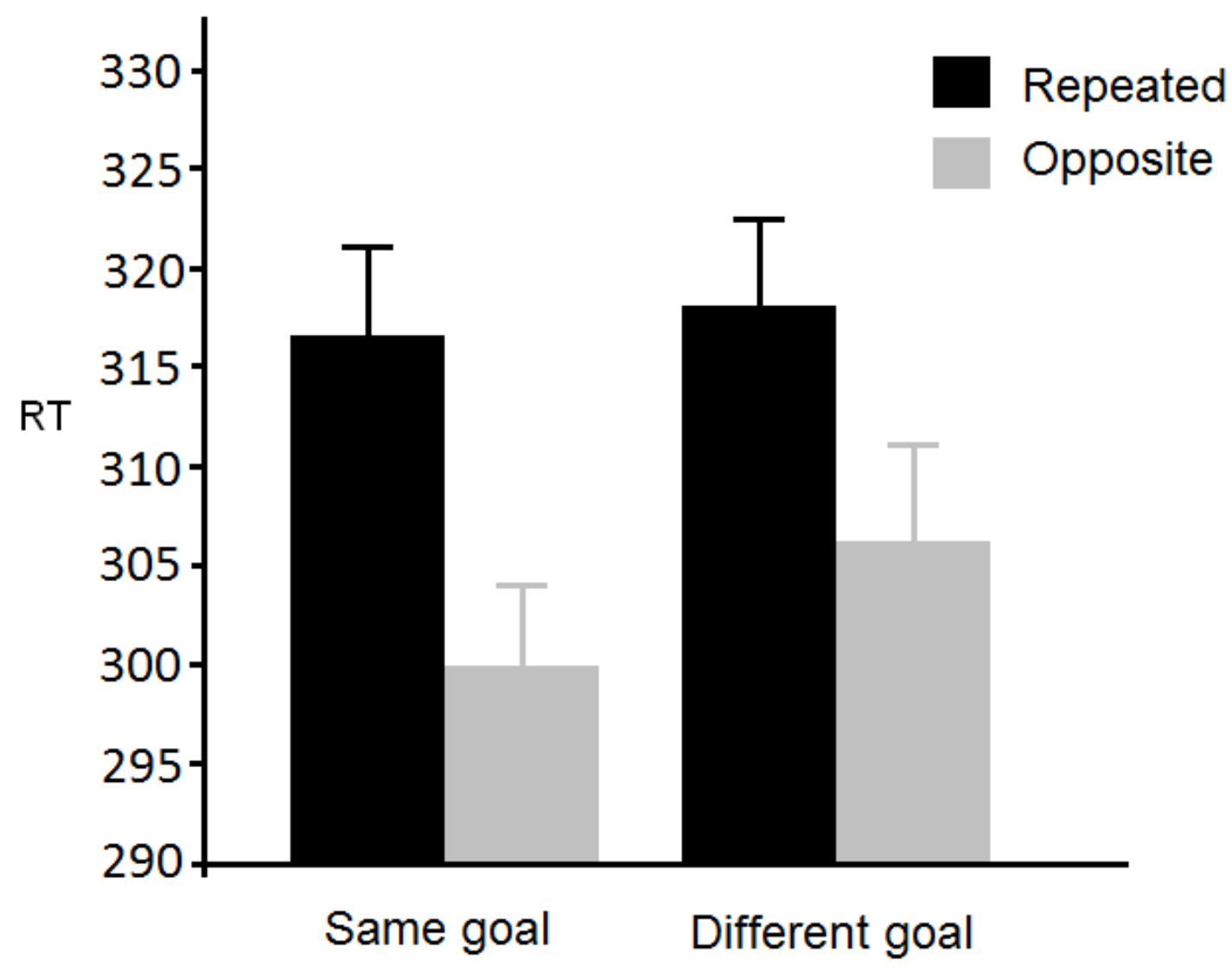

Figure 4 

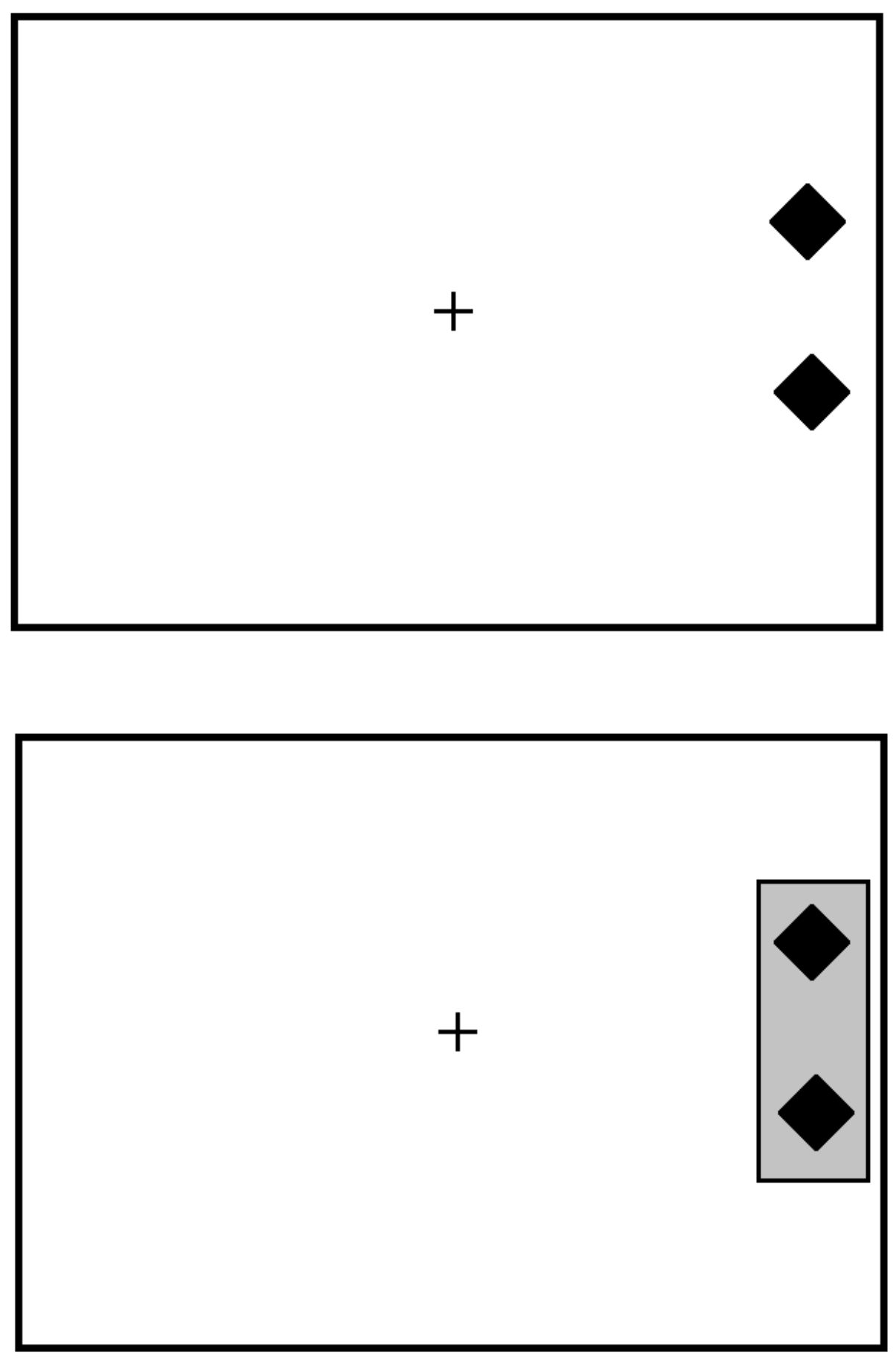

Figure 5 


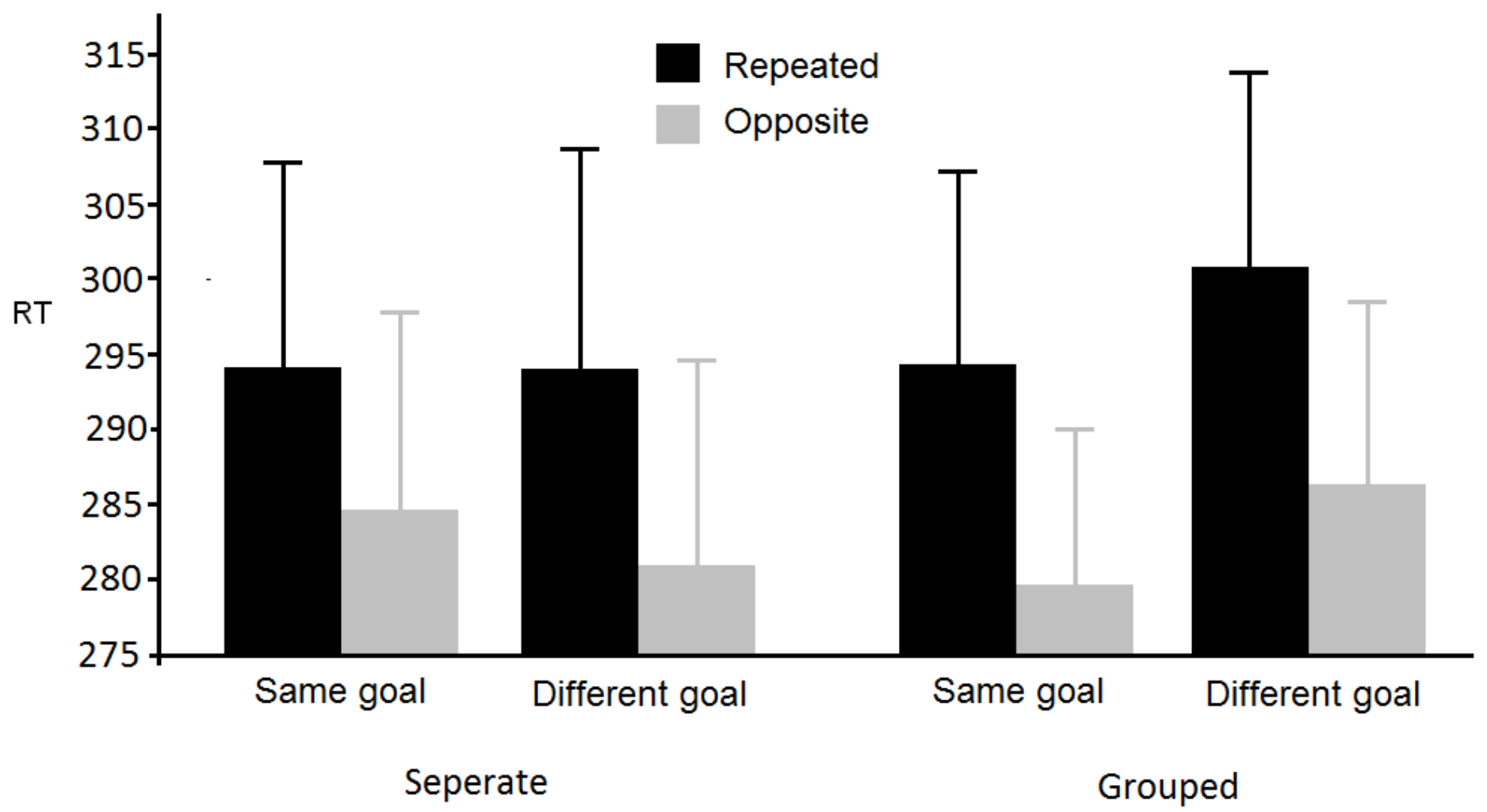

Figure 6 


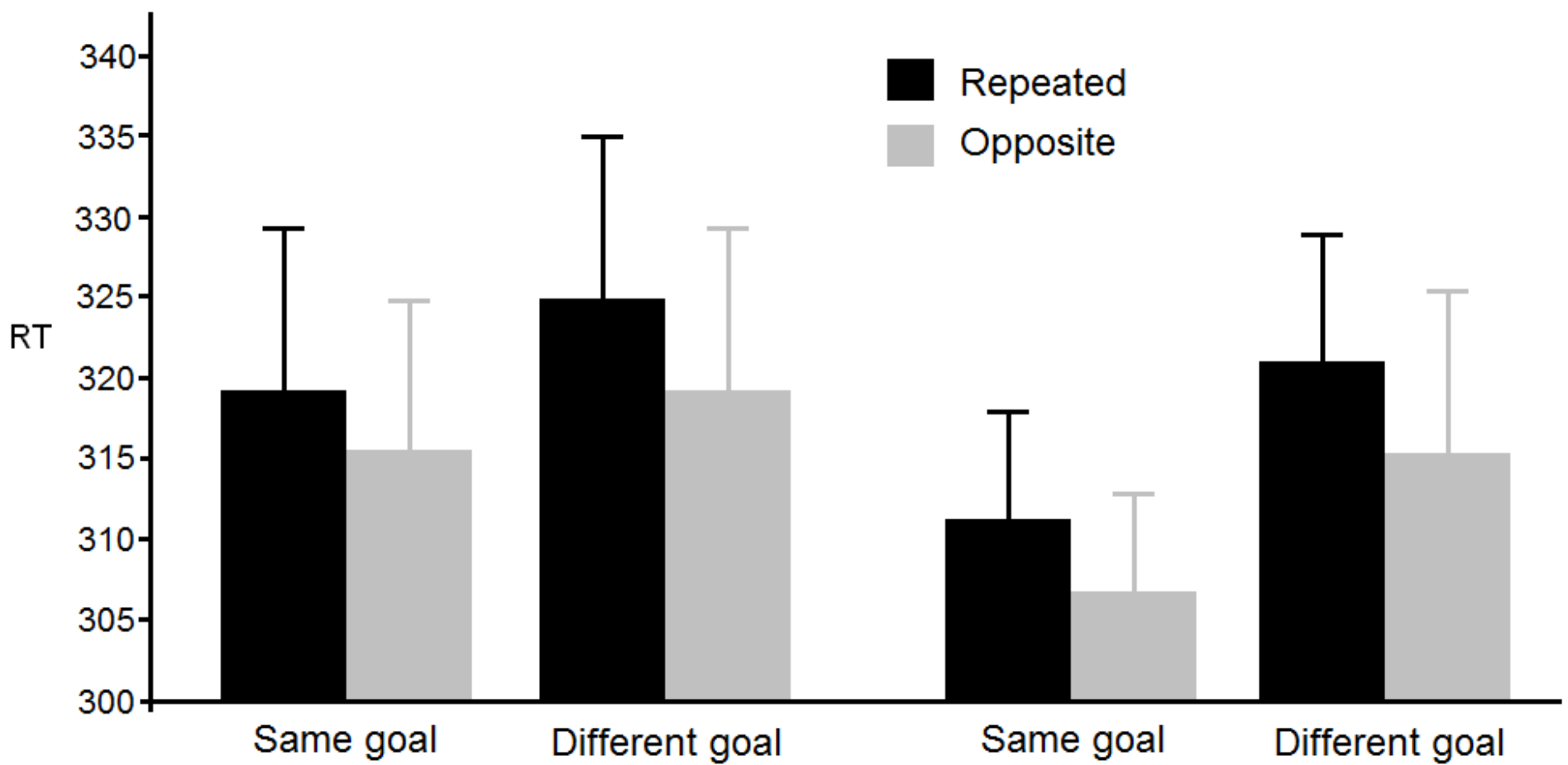

Colour discrimination

Form discrimination

Figure 7 


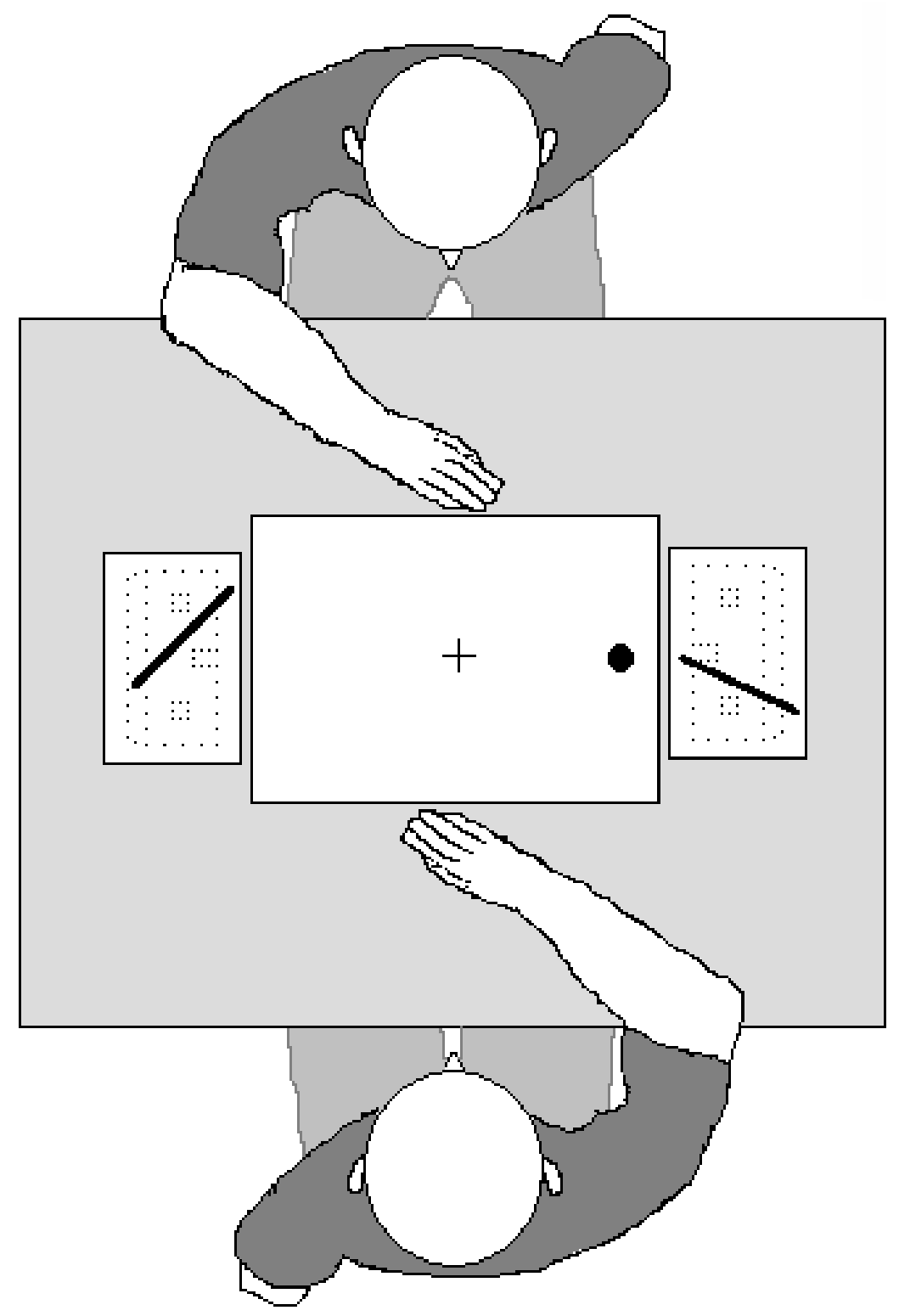

Figure 8 


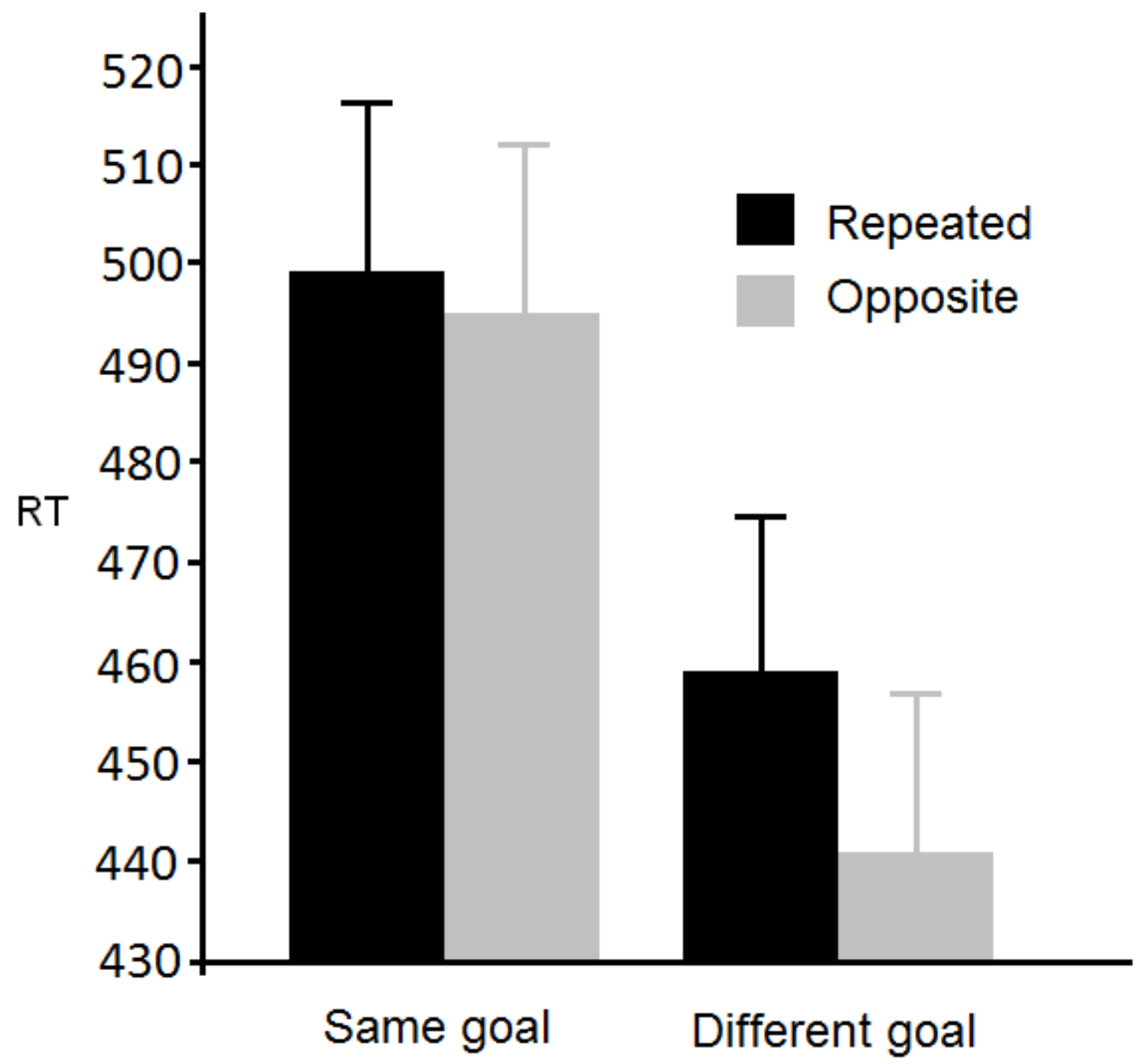

Figure 9 


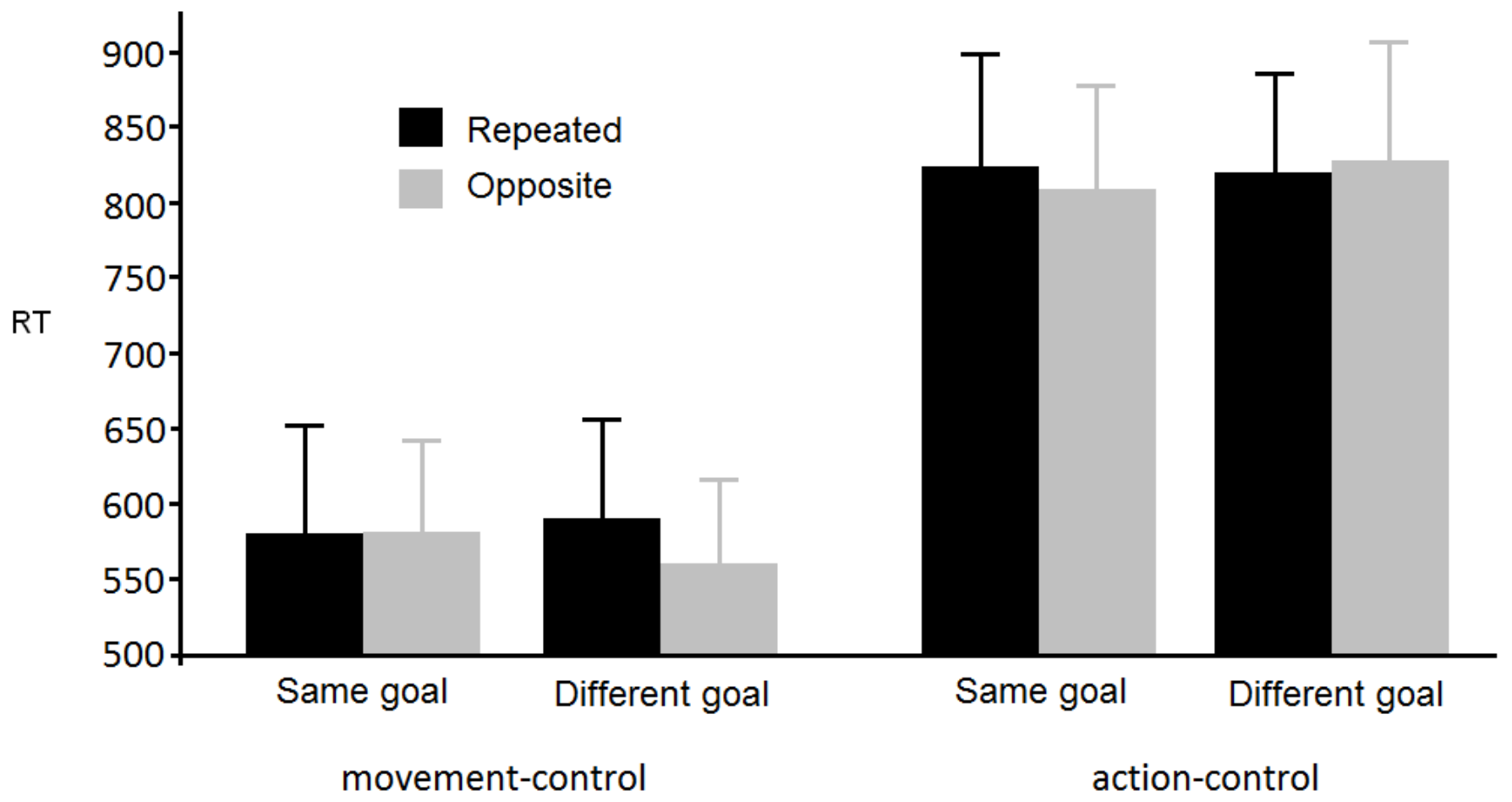

Figure 10 


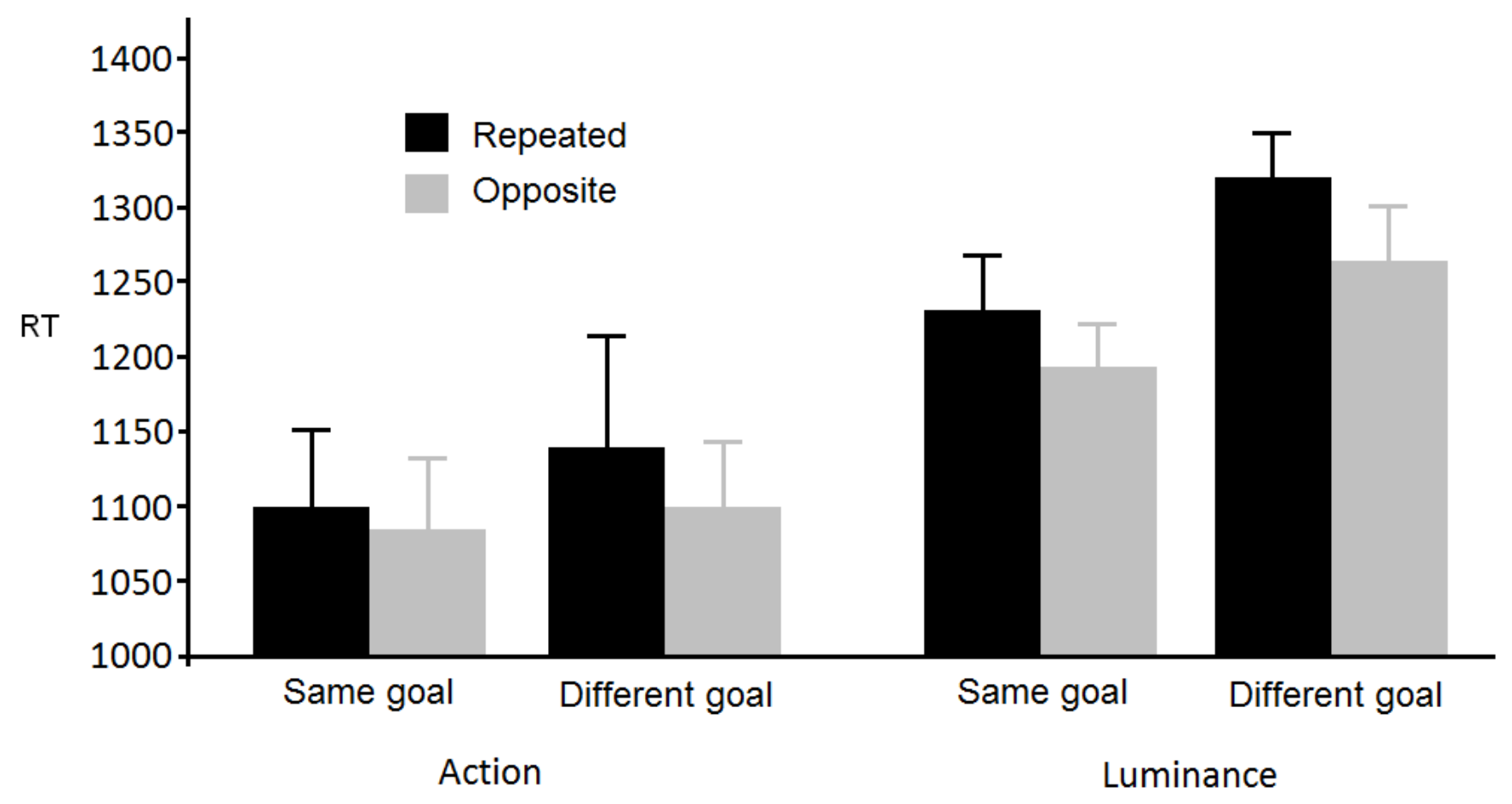

Figure 11 


\begin{tabular}{cccc}
\hline Goal Compatibility & \multicolumn{2}{c}{ Spatial Location } & \\
& Same & Different & Effect Size (ms) \\
\hline Same & $538(87)$ & $517(81)$ & 21 \\
Different & $518(94)$ & $501(87)$ & 17 \\
\hline
\end{tabular}

Table 1

END 